\title{
Longitudinal analysis of antibody dynamics in COVID-19 convalescents reveals neutralizing responses up to 16 months after infection
}

\author{
Yang Yang1,3, Minghui Yang1,3, Yun Peng1,3, Yanhua Liang, ${ }^{1,3}$, Jinli Wei', Li Xing', Liping Guo', \\ Xiaohe Li', Jie Li ${ }^{1}$, Jun Wang ${ }^{1}$, Mianhuan Li', Zhixiang Xu', Mingxia Zhang ${ }^{1}$, Fuxiang Wang ${ }^{1}$, Yi Shi ${ }^{2}{ }^{2}$, \\ Jing Yuan $\mathbb{1}^{1,4} \bowtie$ and Yingxia Liu $\mathbb{1}^{1,4} \bowtie$
}

\begin{abstract}
Elucidating the dynamics of the neutralizing antibody (nAb) response in coronavirus disease 2019 (COVID-19) convalescents is crucial in controlling the pandemic and informing vaccination strategies. Here we measured nAb titres across 411 sequential plasma samples collected during 1-480 d after illness onset or laboratory confirmation (d.a.o.) from 214 COVID-19 convalescents, covering the clinical spectrum of disease and without additional exposure history after recovery or vaccination against SARS-CoV-2, using authentic SARS-CoV-2 microneutralization (MN) assays. Forty-eight samples were also tested for neutralizing activities against the circulating variants using pseudotyped neutralization assay. Results showed that anti-RBD IgG and $M N$ titres peaked at $\sim 120$ d.a.o. and subsequently declined, with significantly reduced nAb responses found in $91.67 \%$ of COVID-19 convalescents ( $\geq 50 \%$ decrease in current $M N$ titres compared with the paired peak MN titres). Despite this decline, majority of the COVID-19 convalescents maintained detectable anti-RBD IgG and MN titres at 400-480 d.a.o., with undetectable neutralizing activity found in $14.41 \%(16 / 111)$ of the mild and $50 \%(5 / 10)$ of the asymptomatic infections at 330-480 d.a.o. Persistent antibody-dependent immunity could provide protection against circulating variants after one year, despite significantly decreased neutralizing activities against Beta, Delta and Mu variants. In conclusion, these data show that despite a marked decline in neutralizing activity over time, $\mathrm{nAb}$ responses persist for up to $480 \mathrm{~d}$ in most convalescents of symptomatic COVID-19, whereas a high rate of undetectable nAb responses was found in convalescents from asymptomatic infections.
\end{abstract}

S evere acute respiratory syndrome coronavirus 2 (SARS-CoV-2), the seventh member of the coronavirus family found to infect humans, was detected in late December 2019 $9^{1,2}$. The SARS-CoV-2 pandemic is ongoing and to date has caused more than 248 million human infections, with more than 5 million fatal cases worldwide (https://covid19.who.int/). In particular, asymptomatic infections account for up to $40 \%$ of all SARS-CoV-2 infections, and silent transmission during the presymptomatic and asymptomatic stages is responsible for more than $50 \%$ of the overall attack rate in COVID-19 outbreaks ${ }^{3-6}$. Therefore, antibody-mediated protective immunity induced either by natural infection or vaccination is of great importance for the control of the COVID-19 pandemic.

Neutralizing antibodies (nAbs) rise rapidly after infection and are maintained for years to decades by long-lived plasma and memory B cells in most acute viral infections, playing vital roles in viral clearance and protection against viral diseases ${ }^{7}$. Studies have shown that the antibody response induced by coronavirus in humans tends to wane over time and varies between types of coronaviruses and the severity of the disease ${ }^{8}$. Seasonal human coronaviruses, such as HCoV-229E, HCoV-OC43, HCoV-NL63 and HCoV-HKU1, have been shown to induce short-term antibody responses, and re-infection with the same seasonal coronavirus occurs frequently 12 months after infection'. However, the two coronaviruses that cause severe disease in humans, including SARS-CoV and the
Middle East Respiratory Syndrome coronavirus (MERS-CoV), induce stronger and more durable antibody responses for up to 3 years ${ }^{8,10-14}$. Recent data from animal models, therapeutic use of neutralizing monoclonal antibodies, and convalescent plasma in a clinical setting suggest a critical role for $n A b s$ in controlling SARS-CoV-2 infection and re-infection ${ }^{15-22}$, while a key question that has not yet been addressed is whether COVID-19 convalescents could maintain long-lasting antibody-mediated protective immunity. Studies have investigated the dynamic change and longevity of the binding and $\mathrm{nAb}$ response in COVID-19 convalescents, and indicated that the $\mathrm{nAb}$ response in COVID-19 convalescents could be maintained for up to one year after the onset of symptoms, accompanied by a decrease in $\mathrm{nAb}$ titres ${ }^{14,23-26}$.

Understanding the accurate long-term antibody dynamics of SARS-CoV-2 infection is hampered by either the limited number of patient cohorts, the short duration of follow-up, or the availability of authentic virus-based detection of the $\mathrm{nAb}$ response in previous studies. Furthermore, possible re-exposure to SARS-CoV-2 in the COVID-19 pandemic area could substantially influence the natural dynamics and longevity of nAb titres in COVID-19 convalescents. Due to the highly efficient control of endemic COVID-19 in China, the Chinese cohort offers an ideal model to evaluate the natural dynamics and longevity of $n A b$ responses after SARS-CoV-2 infection. In this study, we investigated the dynamics and longevity of

'Shenzhen Key Laboratory of Pathogen and Immunity, National Clinical Research Center for infectious disease, State Key Discipline of Infectious Disease, Shenzhen Third People's Hospital, Second Hospital Affiliated to Southern University of Science and Technology, Shenzhen, China. ${ }^{2}$ CAS Key Laboratory of Pathogenic Microbiology and Immunology, Institute of Microbiology, Chinese Academy of Sciences, Beijing, China. ${ }^{3}$ These authors contributed equally: Yang Yang, Minghui Yang, Yun Peng, Yanhua Liang. ${ }^{4}$ These authors contributed equally: Jing Yuan, Yingxia Liu. ${ }^{\circledR}$-mail: 13500054798@139.com; yingxialiu@hotmail.com 
antibody response up to $480 \mathrm{~d}$ after illness onset or laboratory confirmation (d.a.o.) from 214 COVID-19 convalescents across the clinical spectrum without any additional exposure history after recovery or vaccination against SARS-CoV-2, using an authentic virus-based microneutralization assay, and tested the neutralizing activity against circulating variants using SARS-CoV-2 pseudotype neutralization assays.

\section{Results}

Baseline characteristics of the cohort. A total of 214 laboratory confirmed patients with COVID-19 were included in this study evaluating the antibody response: 51 individuals in the severe group, 134 individuals in the mild group, and 29 individuals in the asymptomatic group. The cohort consisted of $46.73 \%$ males, with a median age of 48 years (range 2-79) (Table 1), and the patients in the severe group were significantly older than those in the mild and asymptomatic groups. The body mass index (BMI) in the severe group was significantly higher than in the asymptomatic group. The median interval between illness onset and negative qPCR test for SARS-CoV-2 was $21 \mathrm{~d}$, and a longer duration of viral shedding was found in the severe and mild groups compared with the asymptomatic group. The median duration of hospitalization was 32, 20 and $14 \mathrm{~d}$ for the severe, mild and asymptomatic groups, respectively. Complete blood counts for each patient on the date of the first hospital admission or at the first timepoint showed that the expression of D-dimer, C-reactive protein (CRP), interleukin-6 (IL-6), procalcitonin (PCT) and lactate dehydrogenase (LDH) was significantly higher in the severe and mild groups, while the percentage of lymphocytes (LYM), albumin (ALB) and CD4 count was significantly lower in the severe group. Sequential samples were collected at timepoints between 1 and 480 d.a.o. (the d.a.o. for asymptomatic patients was calculated on the basis of days after laboratory confirmation) on the basis of availability, and no individuals with a history of exposure after discharge or SARS-CoV-2 vaccination were found before the last follow-up (Table 1). A total of 411 plasma samples were collected, with 211 samples collected over $180 \mathrm{~d}$ and 134 samples over $360 \mathrm{~d}$.

Persistent nAb response in COVID-19 convalescents. The immunoglobulin-G (IgG), $\operatorname{IgM}$ and $\operatorname{IgA}$ responses against the receptor-binding domain (RBD) of S glycoprotein and IgG response against $\mathrm{N}$ protein were measured by ELISA over multiple timepoints and $\mathrm{nAb}$ titres were measured using a microneutralization (MN) assay (Fig. 1 and Extended Data Fig. 1). Samples collected between 180 and 330 d.a.o. possessed a $100 \%$ positive rate for anti-RBD IgG and detectable neutralizing activity ( $\mathrm{MN}$ titres $\geq 10$ ) against live SARS-CoV-2 (Fig. 1a,b). However, individuals with undetectable anti-RBD and neutralizing activity were detected during 331 to 480 d.a.o., and positive rates varied from 90.9 to $100 \%$ and 72 to $87.5 \%$, respectively (Fig. 1a,b). In particular, these individuals were mainly in the mild and asymptomatic groups, and the rate of individuals with undetectable neutralizing activity reached $50 \%$ (Fig. 1c). Meanwhile, the distribution of MN titres obtained during 180-480 d.a.o. showed that individuals with $\mathrm{MN}$ titres $\geq 160$ decreased rapidly, while the proportion of $\mathrm{MN}$ titres ranging from negative to 80 increased rapidly over time, indicating a decline in neutralizing activity in COVID-19 convalescents. Notably, overall positive rates of $90.43 \%$ (range $80.95-100 \%$ ) for anti-RBD IgA, 21.53\% (range 10.52-53.85\%) for anti-RBD IgM and $96.68 \%$ (range 90.9-100\%) for anti-N IgG during 180-480 d.a.o. were also found (Fig. 1a and Extended Data Fig. 1a,b). Combining the IgG response and the MN titre measurements, a high coincidence rate of $93.19 \%$ and significant correlations were found between the anti-RBD IgG and MN titres (Fig. 1e,f and Extended Data Fig. 1c), especially the correlations in severe and mild groups (Extended Data Fig. 1d).
Comparison of the $\mathrm{nAb}$ response among COVID-19 patients across the clinical spectrum. Differences in antibody development within $28 \mathrm{~d}$ between the severe, mild and asymptomatic groups (Extended Data Fig. 2) were first analysed. The overall positive rates and expression levels of anti-RBD IgG, anti-RBD IgA, anti-RBD IgM, anti-N IgG and nAbs in the three groups increased with disease progression, and most of these indices showed no significant differences. We then compared the available peak anti-RBD IgG levels and MN titres among the severe, mild and asymptomatic groups. Only individuals in whom the available peak anti-RBD $\operatorname{IgG}(N=46)$ and $\mathrm{MN}$ titres $(N=48)$ occurred before the last timepoint $(\geq 270$ d.a.o.) were included in this analysis (Fig. 2). The highest levels of anti-RBD IgG in the severe and mild groups were comparable, but were significantly higher than in the asymptomatic group (Fig. 2a). Significant differences were found for the current anti-RBD IgG levels among the three groups, with the highest level being observed in the severe group and the lowest in the asymptomatic group (Fig. 2c). Peak MN titres in the severe and mild groups did not show any significant differences, while both were significantly higher than those of the asymptomatic group. In detail, the highest $\mathrm{MN}$ titres for the severe group were all $\geq 80$, and $50 \%$ belonged to the range $640-5,120$. For the mild group, the peak $\mathrm{MN}$ titres ranged from 10 to 1,280 , and $26.06 \%$ belonged to the range $640-5,120$. However, for the asymptomatic group, all maximum $\mathrm{MN}$ titres were $\leq 160$, and $81.82 \%$ belonged to the range $10-80$ (Fig. 2c,e). Regarding current $\mathrm{MN}$ titres, the severe group showed the highest, and no significant difference was found between the mild and asymptomatic groups (Fig. 2d,f). The current MN titres of the severe group mainly belonged to the range $40-320$, while MN titres were negative to 40 for the mild and asymptomatic groups (Fig. 2f). These results indicate that patients with COVID-19 with more severe disease may develop and maintain a stronger $\mathrm{nAb}$ response.

Dynamic change in the $\mathrm{nAb}$ response in COVID-19 convalescents. The general kinetics of anti-RBD IgG, anti-RBD IgM, anti-RBD IgA, anti-N IgG and MN titres in COVID-19 convalescents were analysed (Fig. 3 and Extended Data Fig. 3). Generally, both the anti-RBD IgM and anti-RBD IgA antibodies rose and reached the peak rapidly upon infection. Then anti-RBD IgM decreased rapidly and became negative for most individuals at about 150 d.a.o., while anti-RBD IgA remained detectable for most individuals despite the decrease (Extended Data Fig. 3a,b). Anti-RBD IgG, anti-N IgG and MN titres increased as disease progressed and reached a peak at around 120 d.a.o. (Fig. 3a,b and Extended Data Fig. 3c). Then Anti-RBD IgG and MN titres decreased slowly and remained relatively stable after 400 d.a.o., while anti-N IgG showed a continued decrease. When comparing the three groups, results showed that longer periods were required (about $150 \mathrm{~d}$ ) for the severe group to reach the highest anti-RBD IgG and MN titres than for the mild (about $120 \mathrm{~d}$ ) and asymptomatic (about $80 \mathrm{~d}$ ) groups (Extended Data Fig. 3). To obtain a more precise dynamics of the antibody response of COVID-19 convalescents, detailed dynamic changes in anti-RBD IgG, anti-RBD IgM, anti-RBD IgA, anti-N IgG and $\mathrm{MN}$ titres in patients who got measurements at more than three timepoints during a follow-up of $\geq 300 \mathrm{~d}$ are shown in Fig. 4 . The profiles of both binding and nAb dynamics varied among different individuals. For example, for Patients $01,09,14,19$ and 68, both anti-RBD IgG and nAbs rose and peaked rapidly after infection (within 28 d.a.o.), and although declining, nAbs were still detectable up to $471 \mathrm{~d}$. For Patients 66, 71, 72, 73, 74 and 77, both the anti-RBD IgG and nAbs were detectable within 28 d.a.o. and peaked at about 60 to 90 d.a.o., then decreased but were still be positive up to 477 d.a.o. Conversely, nAbs in Patients 67 and 75 of the mild group, and Patients 86, 193, 195 and 96 of the asymptomatic group became undetectable at $368-470$ d.a.o., although anti-RBD IgG was still detectable in some patients (Patients 68, 186, 193 and 195). 
Table 1 | Baseline characteristics and specimens of COVID-19 cases in this study

\begin{tabular}{|c|c|c|c|c|c|c|c|}
\hline \multirow[t]{2}{*}{ Characteristic } & \multicolumn{4}{|l|}{ COVID-19 cases } & \multicolumn{3}{|c|}{$P$ values ${ }^{\#}$} \\
\hline & Total & Severe (S) & Mild (M) & Asymptomatic (A) & S vs $M$ & S vs $A$ & $M$ vs $A$ \\
\hline & $(N=214)$ & $(N=51)$ & $(N=134)$ & $(N=29)$ & & & \\
\hline Median age (range) & $48(2-79)$ & $62(31-76)$ & $43(2-79)$ & $25(7-58)$ & $<0.001$ & $<0.001$ & $<0.001$ \\
\hline \multicolumn{8}{|l|}{ Age subgroup $(N, \%)$} \\
\hline$<15 \mathrm{yr}$ & $12(5.6)$ & $0(0)$ & $7(5.2)$ & $5(17.2)$ & NS & 0.005 & 0.040 \\
\hline $15-39 y r$ & $74(34.6)$ & $4(7.8)$ & $52(38.8)$ & $18(62.1)$ & $<0.001$ & $<0.001$ & 0.024 \\
\hline $40-59 y r$ & $69(32.2)$ & $15(29.4)$ & $48(36.4)$ & $6(20.7)$ & NS & NS & NS \\
\hline$\geq 60 \mathrm{yr}$ & $59(27.6)$ & $32(62.7)$ & $27(20.1)$ & $0(0)$ & $<0.001$ & $<0.001$ & 0.005 \\
\hline Male $(n, \%)$ & $100(46.73)$ & $31(60.78)$ & $59(44.03)$ & $10(34.48)$ & 0.049 & 0.036 & $N S^{\#}$ \\
\hline BMI & $\begin{array}{l}23.14 \\
(21.05-25.22)\end{array}$ & $\begin{array}{l}24.3 \\
(22.18-26.59)\end{array}$ & $\begin{array}{l}23.05 \\
(21.30-25.11)\end{array}$ & $20.83(17.71-23.23)$ & 0.015 & $<0.001$ & $<0.001$ \\
\hline $\begin{array}{l}\text { Co-existing chronic medical } \\
\text { conditions }(n, \%)\end{array}$ & $68(31.3)$ & $27(52.9)$ & $35(26.1)$ & $6(20.7)$ & 0.001 & 0.005 & NS \\
\hline $\begin{array}{l}\text { Onset to admission, median } \\
\text { days (median, IQR) }\end{array}$ & $3(2-7)$ & $5(3-7)$ & $3(1-6)$ & - & 0.006 & - & - \\
\hline $\begin{array}{l}\text { Onset to antiviral treatment, } \\
\text { median days (median, IQR) }\end{array}$ & $3(1-6)$ & $5(3-8)$ & $3(1-6)$ & $1(1-2)$ & 0.012 & $<0.001$ & $<0.001$ \\
\hline $\begin{array}{l}\text { Onset to qPCR negative for } \\
\text { SARS-CoV-2 (median, IQR) }\end{array}$ & $18(12-26)$ & $21(18-33)$ & $17(13-25)$ & $9(5-15)$ & $<0.001$ & $<0.001$ & $<0.001$ \\
\hline $\begin{array}{l}\text { Duration of hospitalization } \\
\text { (median, IQR) }\end{array}$ & $21(16-30)$ & $32(23-40)$ & $20(16-27.75)$ & $14(12-19)$ & $<0.001$ & $<0.001$ & $<0.001$ \\
\hline \multicolumn{8}{|l|}{$\begin{array}{l}\text { Laboratory results, median } \\
\text { (median, IQR) }\end{array}$} \\
\hline $\operatorname{NEU}\left(\times\left. 10^{9}\right|^{-1}\right)$ & $3.12(2.32-4.08)$ & $3.41(2.29-4.34)$ & $2.68(1.85-3.62)$ & $3.44(2.96-4.3)$ & 0.013 & NS & $<0.001$ \\
\hline $\operatorname{LYM}\left(\times\left. 10^{9}\right|^{-1}\right)$ & $1.36(0.95-1.78)$ & $0.97(0.66-1.28)$ & $1.42(1.13-1.68)$ & $2.13(1.59-2.61)$ & $<0.001$ & $<0.001$ & $<0.001$ \\
\hline $\operatorname{ALB}\left(\mathrm{g} \mathrm{dl}^{-1}\right)$ & $\begin{array}{l}43.4 \\
(40.25-46,15)\end{array}$ & $39.4(38.1-42.6)$ & $43.9(41.9-46.7)$ & $45.3(43.8-46.9)$ & $<0.001$ & $<0.001$ & 0.004 \\
\hline D-dimer $\left(\mu \mathrm{g} \mathrm{ml}^{-1}\right)$ & $0.39(0.24-0.59)$ & $0.56(0.38-1.14)$ & $0.4(0.24-0.57)$ & $0.23(0.22-0.32)$ & $<0.001$ & $<0.001$ & 0.005 \\
\hline $\operatorname{CRP}\left(\mathrm{mgl}^{-1}\right)$ & $9.93(1.87-36.15)$ & $44.7(20.8-76.5)$ & $8.6(3.22-24.52)$ & $0.62(0.25-1.94)$ & $<0.001$ & $<0.001$ & $<0.001$ \\
\hline IL-6 (pg ml-1) & $9.25(2.61-24.47)$ & $\begin{array}{l}29.77 \\
(17.98-50.84)\end{array}$ & $\begin{array}{l}11.77 \\
(4.02-17.57)\end{array}$ & $1.96(1.5-2.94)$ & $<0.001$ & $<0.001$ & $<0.001$ \\
\hline PCT $\left(\mathrm{ng} \mathrm{ml}^{-1}\right)$ & $0.04(0.03-0.07)$ & $\begin{array}{l}0.06 \\
(0.04-0.09)\end{array}$ & $\begin{array}{l}0.04 \\
(0.03-0.05)\end{array}$ & $0.02(0.02-0.03)$ & $<0.001$ & $<0.001$ & 0.013 \\
\hline $\operatorname{LDH}\left(\mathrm{UI}^{-1}\right)$ & $222(178-446.25)$ & $\begin{array}{l}425 \\
(223.5-664.5)\end{array}$ & $\begin{array}{l}227 \\
(182.5-443.5)\end{array}$ & $167(148.5-186.5)$ & $<0.001$ & $<0.001$ & $<0.001$ \\
\hline CD4 (count per ul) & $550(388.5-742)$ & $320(191-423.3)$ & $581(454-764)$ & 779.5 (673.25-981.3) & $<0.001$ & $<0.001$ & 0.003 \\
\hline $\begin{array}{l}\text { Samples collected during } \\
\text { follow-up }(N, \%)\end{array}$ & 411 & $82(19.95)$ & $251(61.07)$ & $78(18.98)$ & - & - & - \\
\hline$\leq 60$ d.a.o. & $116(28.2)$ & $37(45.1)$ & $51(20.3)$ & $28(35.9)$ & - & - & - \\
\hline 61 120 d.a.o. & $65(15.8)$ & $4(4.9)$ & 40 (15.9) & $21(26.9)$ & - & - & - \\
\hline 121 180 d.a.o. & $19(4.6)$ & $3(3.7)$ & $12(4.8)$ & $4(5.1)$ & - & - & - \\
\hline 181 240 d.a.o. & $35(8.5)$ & $2(2.4)$ & $23(9.2)$ & $10(12.8)$ & - & - & - \\
\hline 241 300 d.a.o. & $16(38.9)$ & $0(0)$ & $12(4.8)$ & $4(5.1)$ & - & - & - \\
\hline 301 360 d.a.o. & $26(6.3)$ & $10(12.2)$ & $15(6.0)$ & $1(1.3)$ & - & - & - \\
\hline 361 420 d.a.o. & $73(17.8)$ & $14(17.1)$ & $51(20.3)$ & $8(10.3)$ & - & - & - \\
\hline 421 480 d.a.o. & $61(14.8)$ & $12(14.6)$ & $47(18.7)$ & $2(2.6)$ & - & - & - \\
\hline $\begin{array}{l}\text { Any exposure to SARS-CoV-2 } \\
\text { after discharge }(N, \%)\end{array}$ & $0(0)$ & $0(0)$ & $0(0)$ & $0(0)$ & - & - & - \\
\hline $\begin{array}{l}\text { Vaccination of SARS-CoV-2 } \\
\text { before last follow-up }(N, \%)\end{array}$ & $0(0)$ & $0(0)$ & $0(0)$ & $0(0)$ & - & - & - \\
\hline
\end{tabular}

\#Statistical significance was determined using a two-sided Mann-Whitney U-test. ${ }^{\star} P<0.05,{ }^{\star \star} P<0.01,{ }^{\star \star \star} P<0.001$. NS, not significant. IQR, interquartile range. NEU, neutrophil. 
a

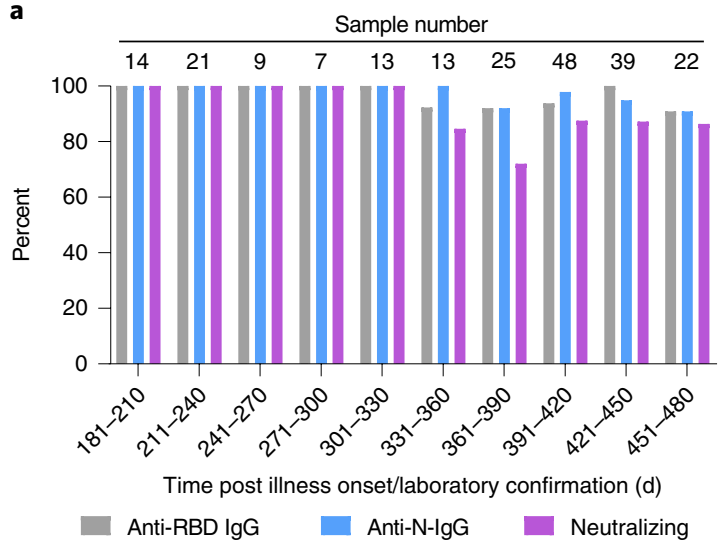

b
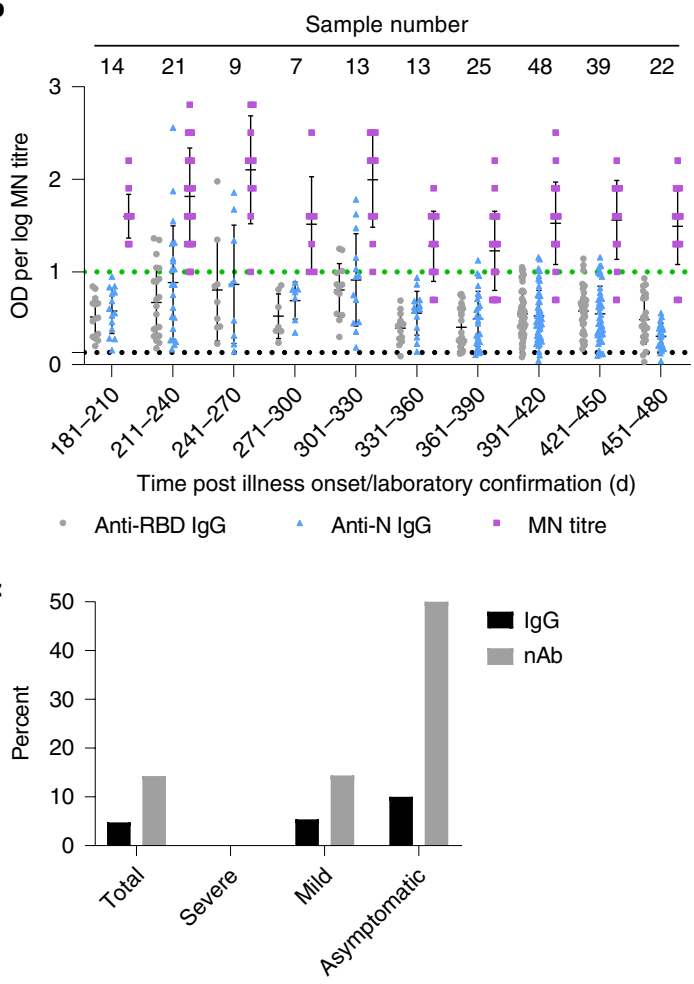

d

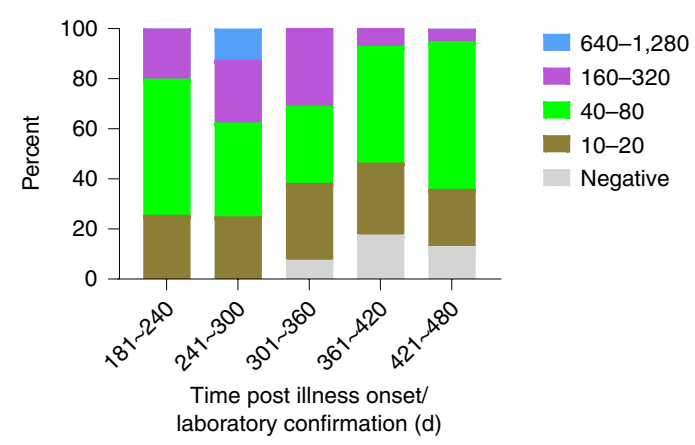

e

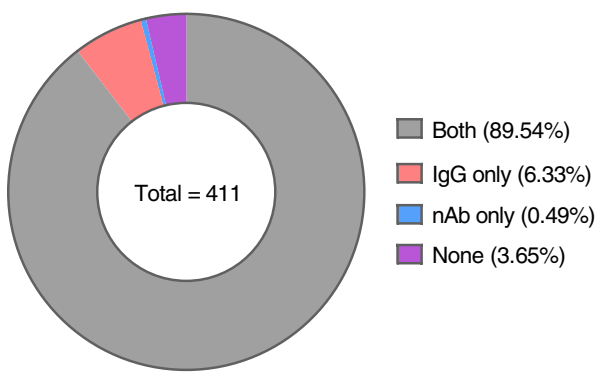

f

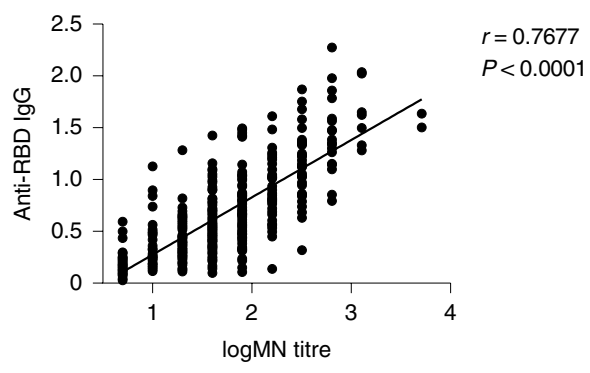

Fig. 1 | Persistent neutralizing antibody responses in COVID-19 convalescents. a, Positive rates of the anti-RBD IgG, anti-N IgG and microneutralization (MN) titres for the 219 plasma samples from 175 COVID-19 convalescents with over $180 \mathrm{~d}$ follow-up. b, Detailed optical density (OD) values of anti-RBD IgG, anti-N IgG and logMN titres of the 219 plasma samples. The line and error bar represent the mean and standard deviation. Black and green dotted lines indicate the limit of detection for anti-RBD IgG and MN titres, respectively. c, Negative rates of anti-RBD IgG and neutralizing activity post 330 d.a.o. d, Dynamic changes in the distribution of MN titres over time. e, Pie chart comparing the fraction of samples with anti-RBD IgG and neutralizing activity. f, Spearman correlation plot between the OD values of anti-RBD IgG and logMN titres. Specimens with MN titres $<10$ were assigned a value of 5 .

Furthermore, a comparison of the anti-RBD IgG levels at the peak and the current anti-RBD IgG levels showed that COVID-19 convalescents experienced a significant decrease in anti-RBD IgG levels, with the median ratio of current/peak levels of $46.38 \%$ (range 13.07-91.99\%) (Fig. 3c,e). A similar pattern was found for the MN titres (Fig. 3d,e), with $91.67 \%$ of COVID-19 convalescents having $\geq 50 \%$ decrease in current $\mathrm{MN}$ titres (Fig. 3f). In particular, stable $\mathrm{MN}$ titres were also found in $8.33 \%$ of the individuals (Fig. $3 \mathrm{f}$ ).

Neutralizing activity against circulating variants of SARS-CoV-2. To test neutralizing efficiency of convalescent plasma against the circulating variants, and compare the potency and breath of plasma samples from the early and the late convalescent phases, we tested and analysed the $50 \%$ inhibitory dose $\left(\mathrm{ID}_{50}\right)$ against the variants wild type (WT), Alpha, Beta, Gamma, Delta, Lambda and Mu using SARS-CoV-2 pseudotype neutralization assays (Fig. 5). A total of 22 and 26 plasma samples were randomly selected from samples with $\mathrm{MN}$ titres $\geq 40$ in the early convalescent phase (within 60 d.a.o.) and late convalescent phase (over 360 d.a.o.), respectively. The basic information is shown in Supplementary Table 1. The results indicate that plasma samples from most individuals in the late convalescent phase could also efficiently neutralize different variants of SARS-CoV-2, although reduced neutralization capacity was found against most of the variants, especially the Beta, Delta and Mu variants. In particular, the lowest neutralizing activities were observed against the $\mathrm{Mu}$ variant, and about $56.25 \%$ (27/48) of the samples 
a

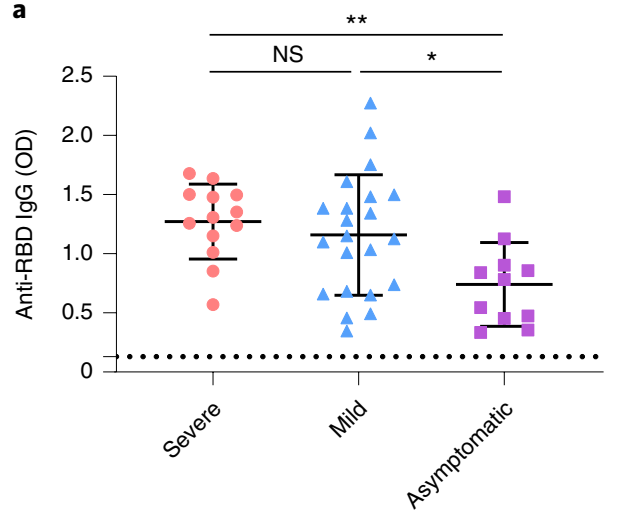

c

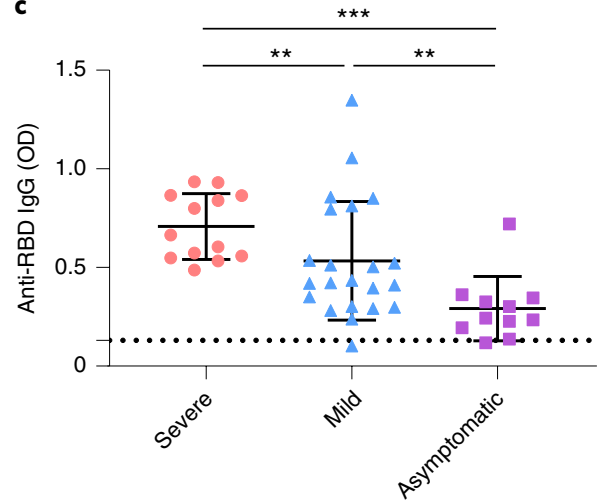

b

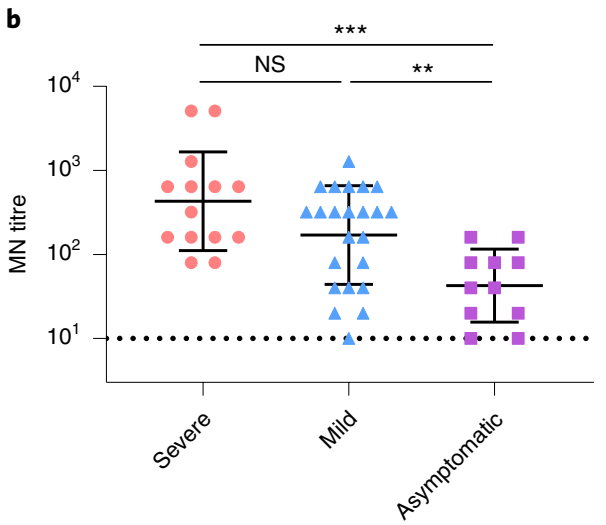

d

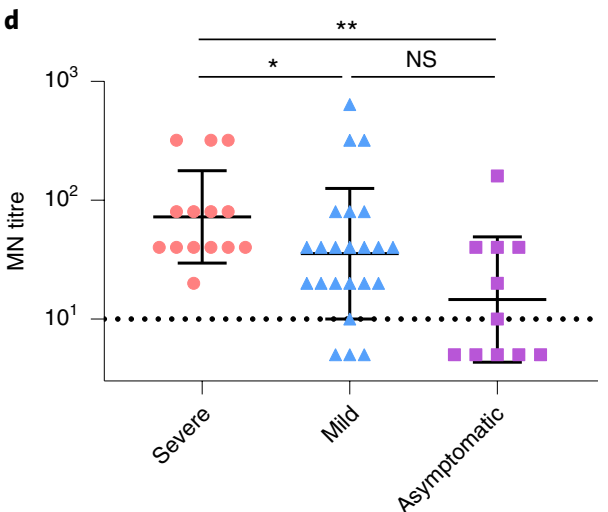

e

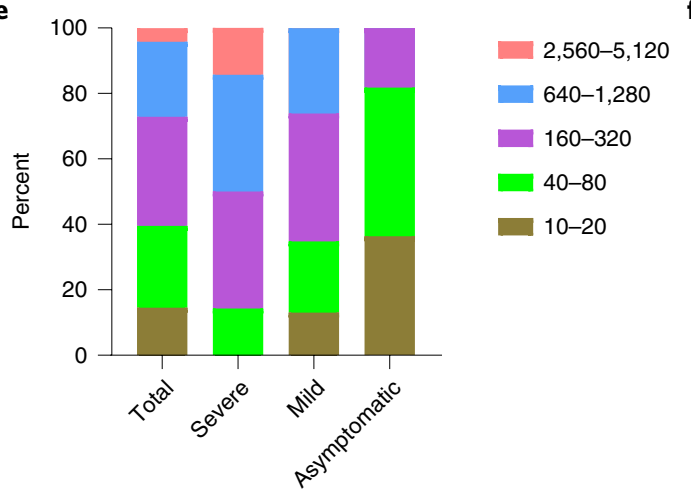

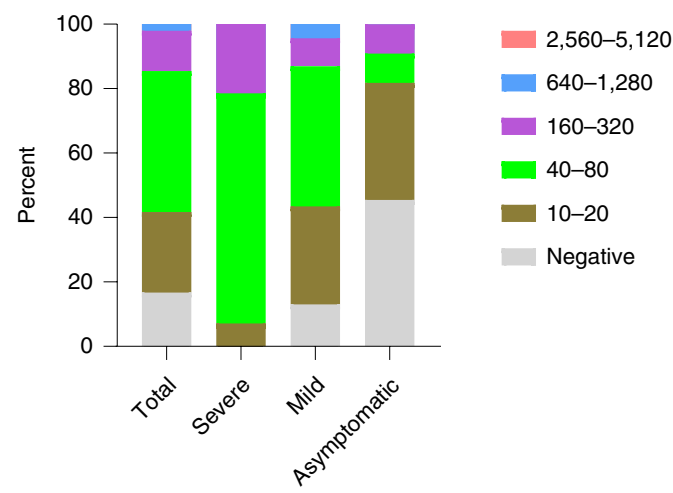

Fig. 2 | Comparison of neutralizing antibody response among different clinical spectra of COVID-19 convalescents. a, Comparison of the available peak levels of anti-RBD IgG for the severe $(n=13)$, mild $(n=22)$ and asymptomatic groups $(n=11)$. P values: severe vs mild, $0.3891 ;$ severe vs asymptomatic, 0.0012; mild vs asymptomatic, 0.0218. Dotted lines indicate the limit of detection. $\mathbf{b}$, Comparison of the available peak MN titres for the severe $(n=14)$, mild $(n=23)$ and asymptomatic groups $(n=11)$. P values: severe vs mild, $0.122 ;$ severe vs asymptomatic, <0.0001; mild vs asymptomatic, 0.0051. c, Comparison of the current levels of anti-RBD IgG for the severe $(n=13)$, mild $(n=22)$ and asymptomatic groups ( $n=11)$. $P$ values: severe vs mild, 0.0061 ; severe vs asymptomatic, $<0.0001$; mild vs asymptomatic, 0.0039 . $\mathbf{d}$, Comparison of the current MN titres for the severe $(n=14)$, mild ( $n=23$ ) and asymptomatic groups $(n=11)$. P values: severe vs mild, 0.0405; severe vs asymptomatic, $0.0014 ;$ mild vs asymptomatic, 0.0714 . Specimens with MN titres $<10$ were assigned a value of 5 . Statistical significance was determined using two-sided Mann-Whitney $U$-test. ${ }^{\star} P<0.05$, ${ }^{\star \star} P<0.01,{ }^{\star \star \star} P<0.001$. The line and error bar represent the mean and standard deviation for the anti-RBD IgG, and geometric mean titre and standard deviation for the MN titres. e,f, Distribution of the available peak and current MN titres for severe, mild and asymptomatic groups.

showed inhibition rates less than $50 \%$ at the first dilution (1:20). Furthermore, the fold reductions in neutralizing activities between the wild-type virus and the Alpha, Beta, Delta and $\mathrm{Mu}$ variants for the samples in the early phase were $2.19,9.28,4.11$ and 13.73, respectively. In the late phase, the fold reductions became 1.26 , 4.74, 3.59 and 7.39 for Alpha, Beta, Delta and Mu variants, respectively, lower than those in the early phase. Representative neutralizing activities against different variants of paired samples from the same patients are shown in Fig. 5b, and a distinct neutralizing pattern was found against the same variant of SARS-CoV-2 between different individuals. For example, paired plasma samples from Patients 1 and 68 showed efficient neutralizing activities against all the variants, while the paired plasma samples from Patients 64, 72 and 203 showed significantly reduced neutralizing activities against Beta, Delta and $\mathrm{Mu}$ variants. Notably, increased neutralizing activities of late phase plasma samples against some variants, such as Delta (Patient 72) and Lambda (Patient 203), were found in some individuals. 
a
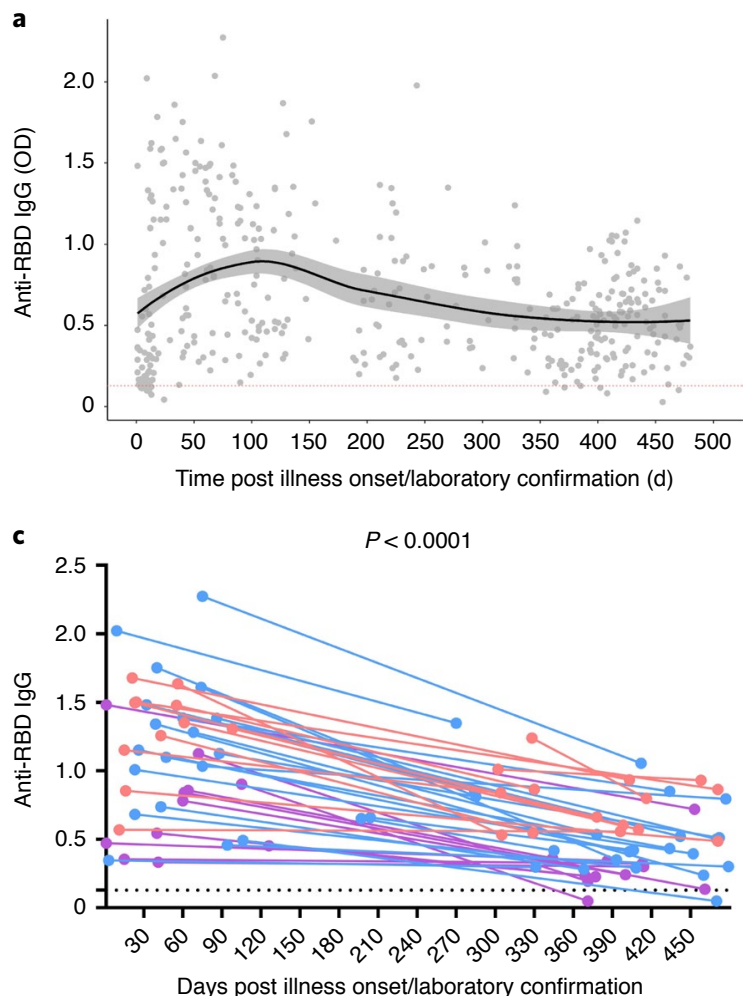

e

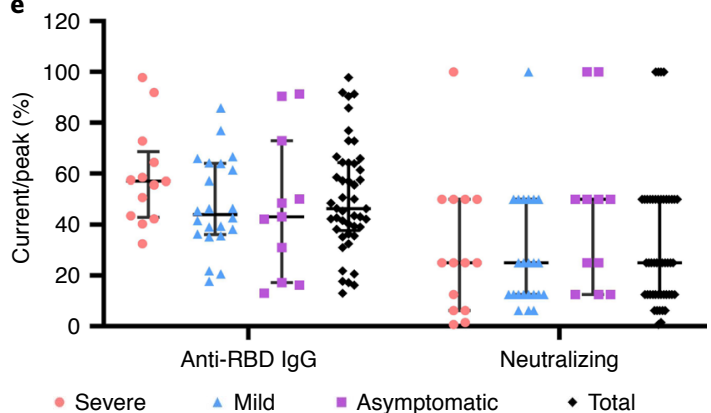

b

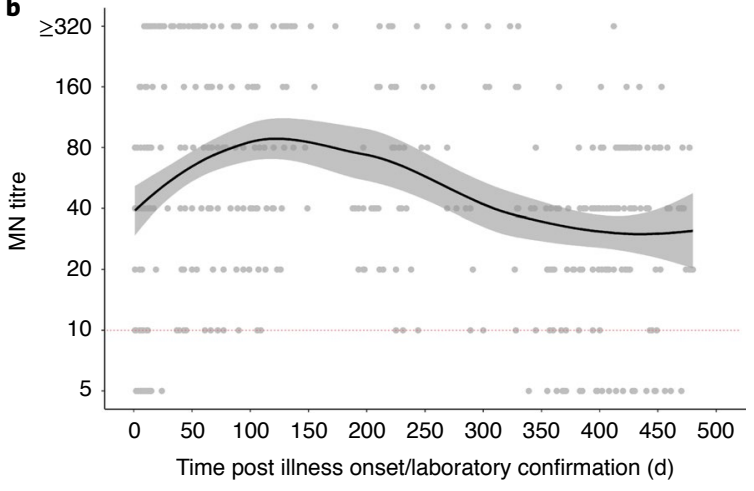

d
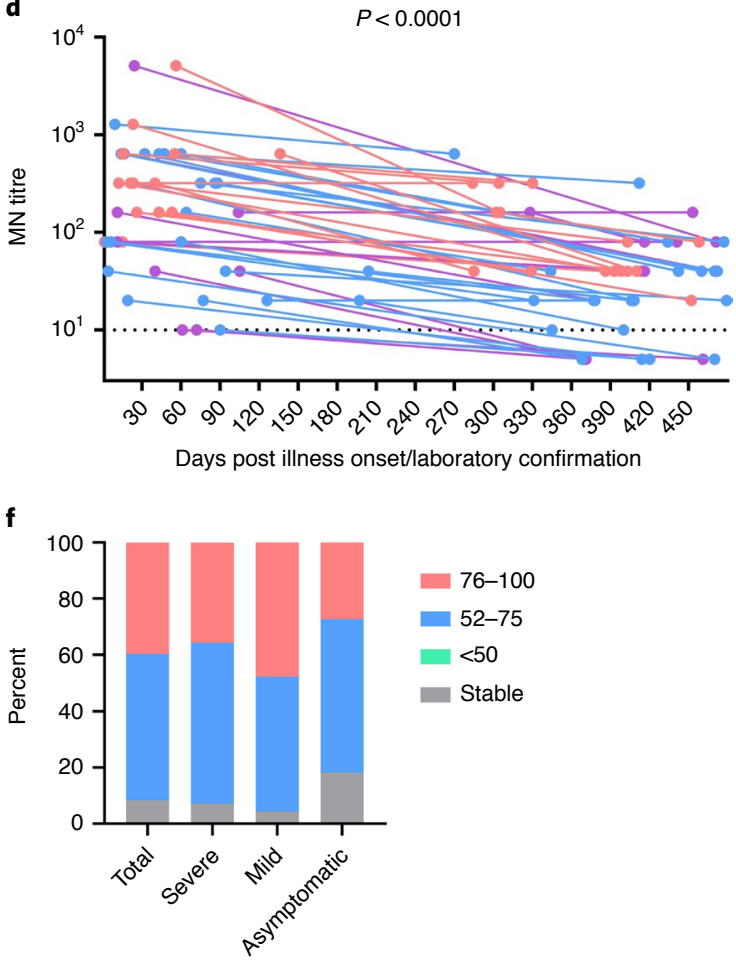

Fig. 3 | Dynamic change of neutralizing antibody response in COVID-19 convalescents. a,b, Kinetics of anti-RBD IgG and MN titres against SARS-CoV-2 during the $480 \mathrm{~d}$ follow-up. The black solid line represents the fitted curve obtained using the LOESS curve-fitting polynomial regression. Grey band areas represent $95 \%$ confidence intervals. c,d, Donor-matched peak and current anti-RBD IgG/MN titres. Only individuals in whom the peak anti-RBD IgG $(N=46)$ and $M N$ titres $(N=48)$ occurred before the last timepoint ( $\geq 270$ d.a.o.) are included in this analysis, and each line represents one individual. Individuals in severe, mild and asymptomatic groups are marked in red, blue and purple, respectively. Statistical significance was determined using a two-sided Mann-Whitney U-test. Dotted lines indicate the limit of detection. e, The ratio of current/peak anti-RBD IgG and MN titres for the three groups. The line and error bar represent the mean and standard deviation. f, Proportion of the different decrease rates in MN titres for the three groups. Specimens with MN titres $<10$ were assigned a value of 5 , and MN titres $\geq 320$ were assigned a value of 320 .

\section{Discussion}

Elucidating the kinetics of neutralizing antibody response in COVID-19 convalescents is crucial for the future control of the COVID-19 pandemic and vaccination strategies. Here we systematically investigated the dynamics and longevity of natural neutralizing antibodies in COVID-19 convalescents, with a combination of the following advantages in our study compared with other studies. First, our study provides the longest follow-up based on a large cohort $(N=214)$ and full clinical spectrum of COVID-19 patients. Second, authentic virus-based microneutralization assay was used to test the neutralizing activity of the plasma samples. Third, all participants were confirmed to be free of re-exposure and vaccination against SARS-CoV-2.
As shown in previous studies, anti-RBD IgM and IgA mainly contribute to the neutralization of SARS-CoV-2 in the early phase of infection, and anti-RBD IgG dominates the neutralization activity of convalescent plasma in the late course of infection and during the convalescent phase ${ }^{27,28}$. Therefore, we focused on the analysis of anti-RBD IgG and nAb responses. Analysis of the dynamics of neutralizing antibodies showed that the anti-RBD IgG and MN titres reached their peak at around 120 d.a.o., then slowly decreased and remained relatively stable after 400 d.a.o. (Fig. 3a,b) in our cohort, which is similar to humoral responses to other viral pathogens ${ }^{7,29}$. The SARS-CoV-2 RBD is immunodominant and accounts for $90 \%$ of the target neutralizing activity present in sera or plasma from most of the evaluated individuals ${ }^{30}$. Given the high coincidence rate 

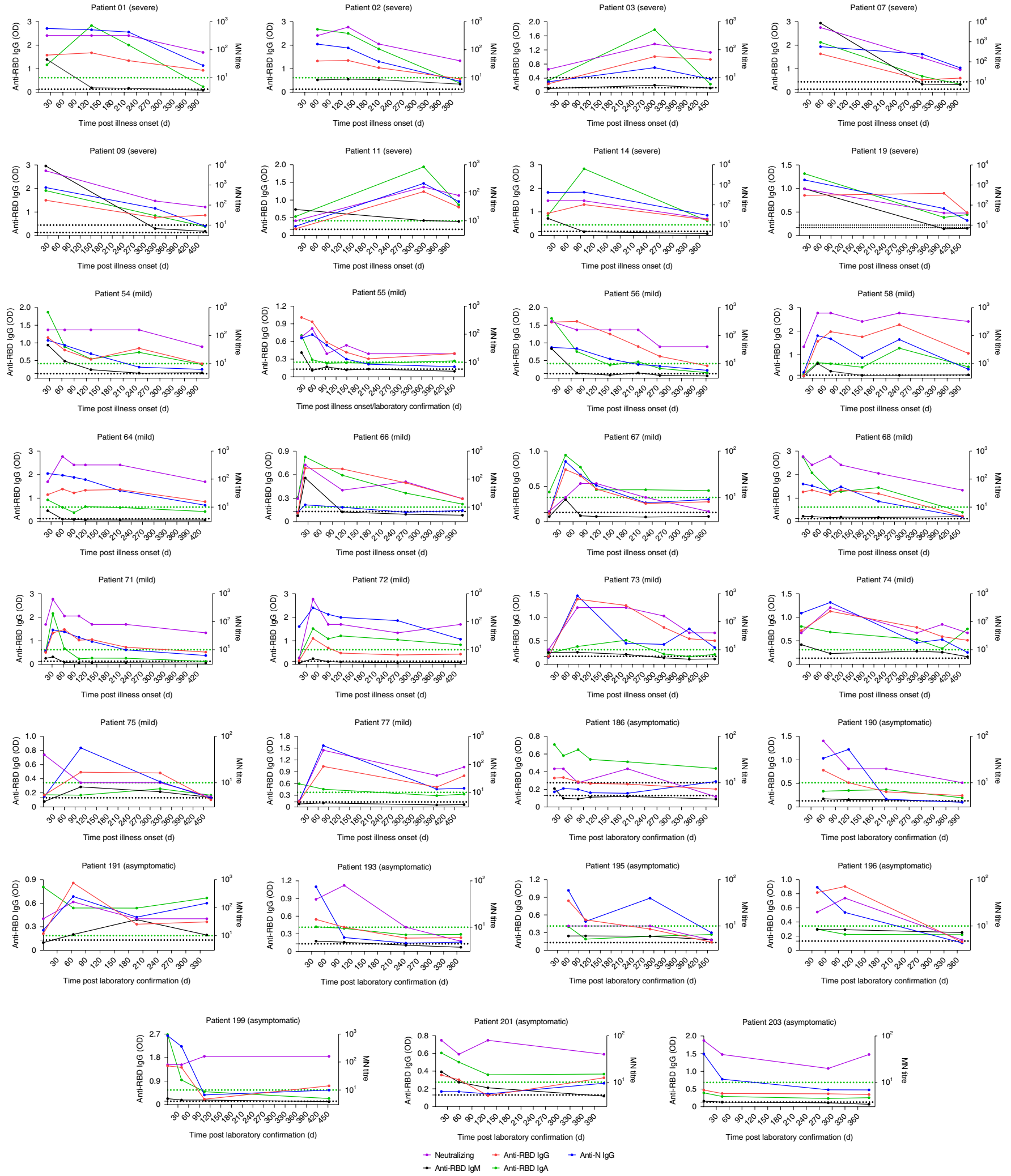

Fig. 4 | Representative dynamic changes in antibody responses (anti-RBD IgG, anti-RBD IgM, anti-RBD IgA, anti-N IgG and MN titres) in 33 COVID-19 patients during acute infection and the convalescent phase. Only patients who got measurements at more than three timepoints with follow-up at day $\geq 300$ are shown. Specimens with MN titres $<10$ were assigned a value of 5. Black and green dotted lines indicate the limit of detection for anti-RBD IgG and $\mathrm{MN}$ titres, respectively.

(93.19\%) and the correlation between anti-RBD IgG levels and MN titres (Fig. 1e,f), the presence of anti-RBD IgG antibodies could also indicate the presence of neutralizing activity in COVID-19 convalescents for most cases. It should be noted that some plasma samples showed high/positive neutralizing activities while having low/negative anti-RBD IgG responses (Figs. 2e and 4), which could 
a
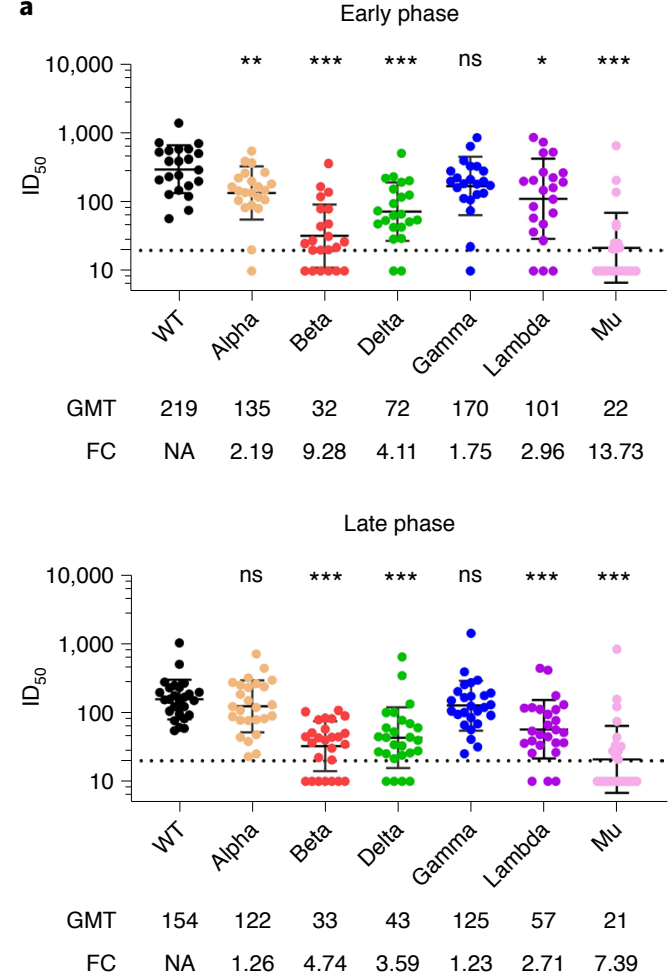

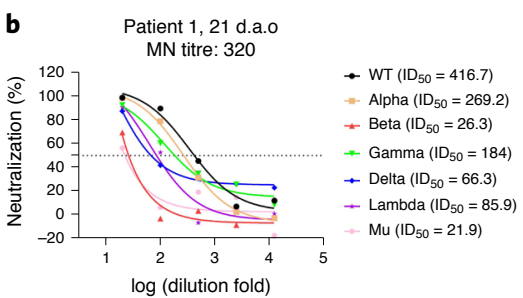

Patient 64, 21 d.a.o MN titre: 320

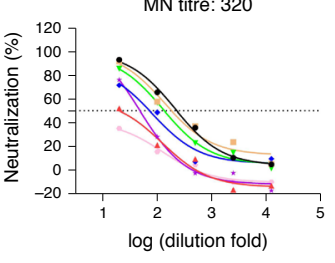

Patient 68, 15 d.a.o MN titre: 640
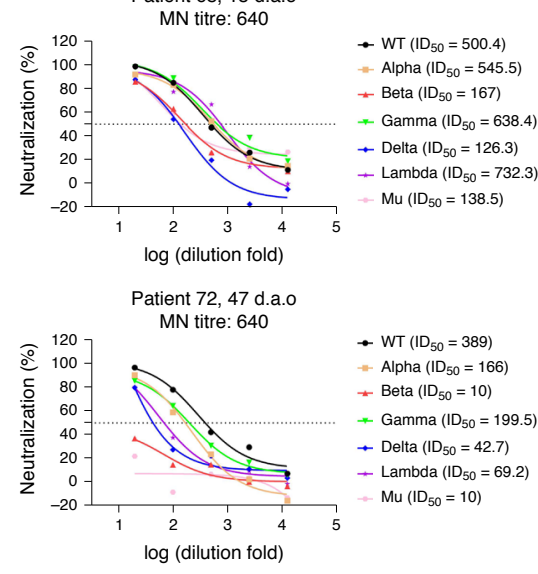

Patient 203, 44 d.a.o MN titre: 40
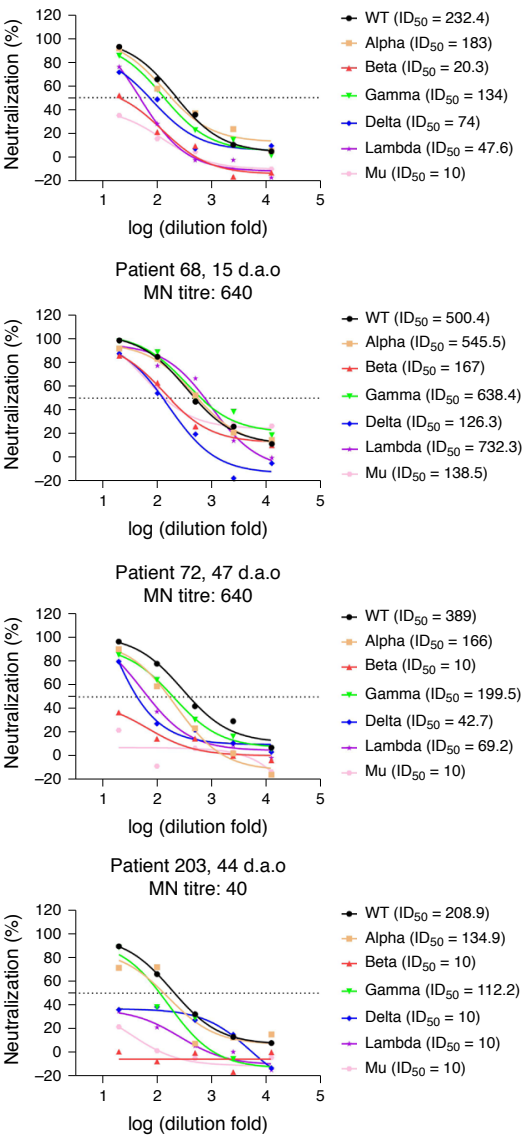

- WT $\left(\mathrm{ID}_{50}=208.9\right)$ Alpha $\left(I_{50}=134.9\right)$

- Beta $\left(\mathrm{ID}_{50}=10\right)$

- Gamma $\left(\mathrm{ID}_{50}=112\right.$

- Delta $\left(\mathrm{ID}_{50}=10\right)$

Lambda $\left(\mathrm{ID}_{50}=10\right)$

$\mathrm{Mu}\left(\mathrm{ID}_{50}=10\right)$

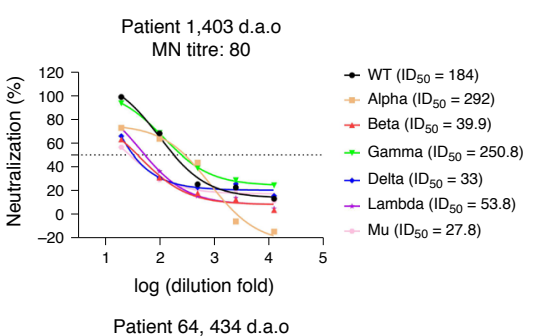

Patient 64, 434 d.a.

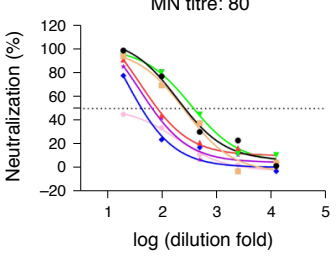

$W T\left(I D_{50}=263.8\right)$ - Alpha $\left(\mathrm{ID}_{50}=248.3\right)$ - Beta $\left(\mathrm{ID}_{50}=82.9\right)$

- Gamma $\left(\mathrm{ID}_{50}=386.2\right)$

- Delta $\left(\mathrm{ID}_{50}=43.5\right)$

Lambda $\left(\mathrm{ID}_{50}=65.9\right)$ $\mathrm{Mu}\left(\mathrm{ID}_{50}=10\right)$

Patient 68,460 d.a.o MN titre: 40

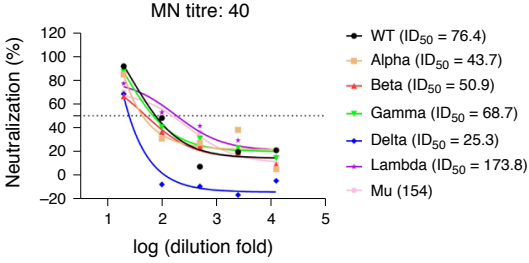

Patient 72, 434 d.a.o

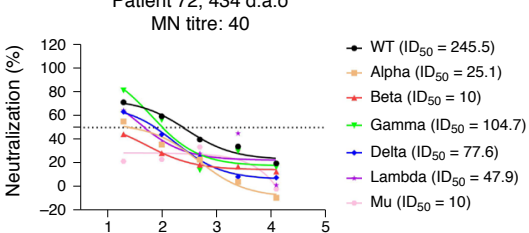

$\log$ (dilution fold)

Patient 203, 386 d.a. MN titre: 40

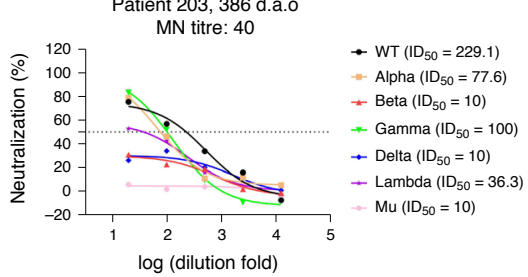

Fig. 5 | Protection of neutralizing antibody from convalescents of COVID-19 against the circulating variants of SARS-CoV-2. a, Neutralizing antibody titres (indicated as $\left(D_{50}\right.$ ) against the WT, Alpha, Beta, Gamma, Delta, Lambda and Mu variants measured at early (between 15 and 60 d.a.0., $N=22$ ) and late (over 360 d.a.o., $N=26$ ) convalescent phases. Black dotted lines indicate the limit of detection for $I D_{50}$. The line and error bar represent the geometric mean titres (GMTs) and the standard deviation for the GMTs. Statistical significance was determined using a two-sided Mann-Whitney U-test. ${ }^{\star} P<0.05,{ }^{\star \star} P<0.01,{ }^{\star \star \star} P<0.001$. $P$ values for the early phase: WT vs Alpha, 0.0028; WT vs Beta, $<0.001 ;$ WT vs Delta, $<0.001 ;$ WT vs Gamma, 0.0543; WT vs Lambda, 0.0108; WT vs Mu, <0.001. P values for the late phase: WT vs Alpha, 0.4318; WT vs Beta, <0.001; WT vs Delta, <0.001; WT vs Gamma, 0.3281 ; WT vs Lambda, $<0.001$; WT vs Mu, $<0.001$. Specimens with ID $D_{50}<20$ were assigned a value of 10 . GMTs and fold changes (FCs) of ID $D_{50}$ between the variants and the wild-type SARS-CoV-2 are shown at the bottom. $\mathbf{b}$, Representative neutralizing activities against different variants of the paired samples from the same patients in both the early and the late convalescent phases. Patient numbers, MN titres and follow-up days are shown at the top of each graph.

result from the contribution of $\operatorname{IgM}$, IgA and antibodies targeting domain A or quaternary epitopes in the S trimer or the $\mathrm{S} 2$ subunit, or from the ongoing affinity maturation of the antibody response $\mathrm{e}^{27,28,31}$. It is well characterized that the magnitude of the antibody response against SARS-CoV-2 is correlated with COVID-19 severity ${ }^{26,31-33}$. Our results also showed that the peak and current anti-RBD IgG and $\mathrm{MN}$ titres obtained were highest in the severe group, followed by the mild group and then the asymptomatic group. It is noteworthy that the severe group started with higher levels of anti-RBD IgG and MN titres than the mild and asymptomatic groups, but it took longer to reach the maximum anti-RBD IgG and MN titres (Extended Data Fig. 2). This might result from the different levels of antigenic exposure due to the higher viral load and prolonged viral shedding in severely infected patients ${ }^{32,34}$.

SARS-CoV-2 re-infection and vaccine breakthrough have now been frequently reported months after initial infection or vaccination $^{35-41}$, suggesting that antibody-mediated immunity may not confer sterilizing immunity, especially in seropositive individuals with lower amounts of baseline SARS-CoV-2 antibody ${ }^{38}$. A key question to address is what level of the $\mathrm{nAb}$ response could provide protection against infection or re-infection. Several large-cohort studies have shown that antibody-mediated immunity could provide strong protection against re-infection in seropositive individuals for up to 7 months after infection, with protection efficacy ranging from $80.5 \%$ 
to $95 \%{ }^{30,42,43}$. A recent study suggested that the estimated neutralization level of $50 \%$ protection against detectable SARS-CoV-2 infection was $20.2 \%$ of the mean convalescent level and the estimated neutralization level required for $50 \%$ protection against severe infection was approximately $3 \%$ of the mean convalescent level ${ }^{44}$. In this context, the $\mathrm{nAb}$ response would be sufficient to provide protection against re-infection and severe infection at 480 d.a.o. (Fig. 1a) for most individuals according to our study. However, SARS-CoV-2 caused mainly asymptomatic to mild infections, and asymptomatic infection constituted up to $40 \%$ of all infections ${ }^{6}$. Due to the high rate of undetectable $\mathrm{nAb}$ response $(14.41 \%$ for the mild group and $50 \%$ for the asymptomatic group) after $330 \mathrm{~d}$, timely detection and vaccination could be necessary to maintain antibody-mediated protection against SARS-CoV-2 for such individuals.

The $S$ protein, particularly the RBD serves as the main target of neutralizing antibodies generated by SARS-CoV-2 infection and vaccination $^{45,46}$. Mutations in S proteins occurred frequently during natural global transmission or under immune selection pressure, and some key mutations have been found to influence the binding between $S$ protein and its receptor, or mediate immune escape against monoclonal antibodies or convalescent plasma ${ }^{47-53}$. Fortunately, recent studies have shown that the currently licensed and widely used inactivated whole virus or mRNA vaccines targeting RBD are still effective against circulating variants, although partial resistance was observed ${ }^{54-59}$. In our study, all patients were admitted to our hospital from 11 January to 18 April 2020, most probably infected with wild-type SARS-CoV-2 without the prevalent key mutations. Our results showed that antibody-dependent immunity could also provide protection against most circulating variants during a $480 \mathrm{~d}$ follow-up, although significantly decreased neutralizing activities against Beta, Delta and $\mathrm{Mu}$ variants were found (Fig. 5). Further, the fold changes in the neutralizing activities between the wild-type virus and the potent variants of immune escape-Beta, Delta and $\mathrm{Mu}$-decreased in the late convalescent phase (Fig. 5). This is consistent with recent studies showing that antibodies evolve in convalescent patients due to the maturation of memory B cells, resulting in an increase in the breadth and potency of specific antibodies for SARS-CoV-2 ${ }^{60-62}$. It is worth noting that plasma samples from different COVID-19 convalescents showed a distinct neutralizing pattern against the same circulating variants of SARS-CoV-2 as observed in our study (Fig. 5) and previous studies ${ }^{31,56,63}$. Several aspects, including different levels of antigenic exposure, affinity maturation of neutralizing antibodies, and expanded clones of viral-antigen-binding B cells could contribute to this heterogeneity of antibody responses in different COVID-19 convalescents $^{60,64}$

Although re-infection occurred in COVID-19 convalescents or vaccinees, symptomatic re-infections and severe diseases occurred at a lower rate than in primary infections ${ }^{39,40,42,65}$. Consequently, whether COVID-19 convalescents need to receive vaccination and when, and whether the former vaccinees need to receive revaccination and when, are of clinical concern. Some recent studies have suggested that both the binding and nAb responses had a similar rate of decline between vaccination with the mRNA vaccine and infection during the first 7 months after vaccination ${ }^{66,67}$. Furthermore, individuals previously diagnosed with COVID-19 could develop rapid humoral immunity after a single dose of the mRNA vaccine ${ }^{68-70}$. In our study, all individuals in the cohort did not receive SARS-CoV-2 vaccination before the current last follow-up, while many individuals started receiving the inactivated SARS-CoV-2 vaccine. Given the extensive use of inactivated SARS-CoV-2 vaccine in over 40 countries, further datasets from this cohort and a naive control group would be valuable in furthering our understanding of how immune responses differ between convalescence and vaccination, and how we can improve the latter with relevant booster shots or updated formulations.
In conclusion, this study provides more precise and comprehensive understanding of the natural antibody response of SARS-CoV-2 infection. The results show that despite a marked decline in neutralizing activity over time, $\mathrm{nAb}$ responses persist for up to $480 \mathrm{~d}$ in most convalescents of symptomatic COVID-19, whereas a high rate of undetectable $\mathrm{nAb}$ responses was found in convalescents from asymptomatic infections.

\section{Methods}

Patient information and sample collection. Participants presented in this study were laboratory confirmed COVID-19 patients using quantitative real-time PCR (Mabsky Biotech Co., Ltd. and ShenZhen ZiJian Biotech Co., Ltd.) and were admitted to our hospital during 11 January to 18 April $2020(N=214)$. These individuals were further divided into severe (including critically ill and severe patients), mild (including mild and moderate patients) and asymptomatic groups on the basis of disease severity categorized according to the China National Health Commission Guidelines for Diagnosis and Treatment of SARS-CoV-2 infection. Clinical information and laboratory results were collected at the earliest timepoint after hospital admission. Blood samples were collected from the enrolled patients during hospitalization and follow-up. The study protocol was approved by the Ethics Committees of Shenzhen Third People's Hospital (2020-010). Written informed consent was obtained from all patients. The study was conducted in accordance with the International Conference on Harmonization Guidelines for Good Clinical Practice, the Declaration of Helsinki and institutional ethics guidelines.

\section{Disease severity classification. Disease severity classification was evaluated} according to the China National Health Commission Guidelines for Diagnosis and Treatment of SARS-CoV-2 infection (seventh version) as previously reported ${ }^{71,72}$. In brief, laboratory confirmed patients with fever, respiratory manifestations and radiological findings indicative of pneumonia were considered moderate cases. Laboratory confirmed patients who met any of the following were considered to have severe COVID-19: (1) respiratory distress (respiration rate $\geq 30 \mathrm{~min}^{-1}$ ), (2) resting oxygen saturation $\leq 93 \%$, or (3) arterial oxygen partial pressure $\left(\mathrm{PaO}_{2}\right) /$ fraction of inspired oxygen $\left(\mathrm{FiO}_{2}\right) \leq 300 \mathrm{mmHg}(1 \mathrm{mmHg}=0.133 \mathrm{kPa})$. Laboratory confirmed patients who had any of the following were considered critically ill: (1) respiratory failure requiring mechanical ventilation, (2) shock, or (3) failure of other organs requiring intensive care unit (ICU). The asymptomatic cases were defined as previously reported ${ }^{73}$.

Cell lines and viruses. African green monkey kidney Vero cells (ATCC, CCL-81) were obtained from ATCC, and 293 cells stably expressing ACE-2 (293-ACE-2) were obtained from Vazyme (Vazyme Biotech Co., Ltd.). Both cell lines were maintained in Dulbecco's minimal essential medium (DMEM) (GIBCO) supplemented with $10 \%$ foetal bovine serum (FBS) (Corning) and penicillin $\left(100 \mathrm{U} \mathrm{ml}^{-1}\right)$-streptomycin $\left(100 \mathrm{mg} \mathrm{ml}^{-1}\right)(\mathrm{GIBCO})$. A clinical isolate of SARS-CoV-2 named BetaCoV/ Shenzhen/SZTH-003/2020 (EPI_ISL_406594) was isolated from the bronchoalveolar lavage fluid sample of the COVID-19 patients using Vero cells in a biosafety level 3 (BSL-3) laboratory ${ }^{74}$. The $50 \%$ tissue culture infectious dose $\left(\mathrm{TCID}_{50}\right)$ assay was done to measure the titres of the viral stock as previously described ${ }^{75,76}$. In brief, Vero cells in 96-well plates were grown to $90 \%$ confluence and infected with 10 -fold serial dilutions of the viral stock for $1 \mathrm{~h}$ at $37^{\circ} \mathrm{C}$. Then the cells were overlaid with fresh DMEM supplemented with $2 \%$ FBS. At $4 \mathrm{~d}$ post infection, plates were assessed for the lowest dilution in which $50 \%$ of the wells exhibited cytopathology. The TCID $_{50}$ values were calculated according to the Reed-Muench method ${ }^{77}$.

Viral entry inhibition assay with pseudotyped SARS-CoV-2 virus. SARS-CoV-2 pseudotype neutralization assays were conducted to test the neutralizing activity of plasma from COVID-19 convalescents using pseudotyped HIV virus incorporated in different variants of SARS-CoV-2 S proteins (Vazyme Biotech Co., Ltd.) as previously reported with some modification ${ }^{78}$. Plasma samples were heat-inactivated at $56^{\circ} \mathrm{C}$ for $30 \mathrm{~min}$ to remove complement activity. Then samples were serially diluted in 5-folds with DMEM supplemented with $2 \%$ FBS from 1:20 to $1: 12,500$ in white, flat-bottom 96-well plates (Thermo Fisher) in a total volume of $50 \mu \mathrm{l}$. Then $200 \mathrm{TCID}_{50}$ of SARS-CoV-2 pseudotyped particles in $50 \mu \mathrm{l}$ were added to each well and incubated at $37^{\circ} \mathrm{C}$ for $1 \mathrm{~h}$. A total of $4 \times 10^{5}$ ACE-2-293T cells in $100 \mu \mathrm{l}$ complete media were added per well and incubated for $48 \mathrm{~h}$ at $37^{\circ} \mathrm{C}$ and $5 \% \mathrm{CO}_{2}$. Firefly luciferase activity (luminescence) was measured using Bright-Light Luciferase Assay System (Vazyme Biotech Co., Ltd.) and a VARIOSKAN LUX Multi-Mode plate reader (Thermo) according to the manufacturers' protocols. Measurements were performed in triplicate and relative luciferase units were then converted into neutralization percentages. Neutralizing antibody titres were calculated as $\mathrm{ID}_{50}$ expressed as the dilution of plasma that resulted in a $50 \%$ reduction of luciferase luminescence compared with a virus control in single-round pseudovirus infection assay as previously reported ${ }^{79}$.

Authentic SARS-CoV-2 microneutralization (MN) assays. Authentic SARS-CoV-2 microneutralization assays were performed as described previously ${ }^{79-81}$. Briefly, 
plasma samples were heat-inactivated at $56^{\circ} \mathrm{C}$ for $30 \mathrm{~min}$ to remove complement activity and then serially diluted in 2-folds with DMEM supplemented with $2 \%$ FBS from 1:10 to 1:5,120. Diluted plasma samples were then mixed with 100 TCID $_{50}$ of SARS-CoV-2 and incubated at $37^{\circ} \mathrm{C}$ for $1 \mathrm{~h}$. The mixture was added to Vero cells and incubated at $37^{\circ} \mathrm{C}$ and $5 \% \mathrm{CO}_{2}$ for another $96 \mathrm{~h}$. Then cytopathic effect was evaluated and recorded using microscopy. The $\mathrm{MN}$ antibody titre was determined as the highest dilution with $50 \%$ inhibition of cytopathic effect. Negative controls were plasma samples from healthy donors, and the positive control was SARS-CoV-2 RBD-specific antibody B38, which has been proven to significantly neutralize SARS-CoV-2 in our previous study ${ }^{82}$.

Enzyme-linked immunosorbent assay (ELISA). RBD- and nucleocapsid (N)-specific-binding IgG antibodies were measured using commercial kits from Sino Biological, and RBD-specific-binding IgM and IgA antibodies were measured using commercial kits from Vazyme, according to the manufacturer's protocols. In brief, all plasma samples were heat-inactivated at $56^{\circ} \mathrm{C}$ for $30 \mathrm{~min}$ before use. The plasma samples diluted in 5\% BSA were added to the high-binding ELISA plates pre-coated with antigen (N protein or RBD) and incubated for $2 \mathrm{~h}$ at room temperature. Wells were then washed with PBS-T (PBS with $0.05 \%$ Tween-20), and secondary antibody was added and incubated for $1 \mathrm{~h}$ at room temperature. After another three washes with PBS-T, tetramethylbenzidine substrate was added at room temperature in the dark. After $15 \mathrm{~min}$, the reaction was stopped with a $2 \mathrm{M}$ $\mathrm{H}_{2} \mathrm{SO}_{4}$ solution and then the absorbance was measured at $450 \mathrm{~nm}$.

Statistical analysis. Mann-Whitney $U$-test was used to compare the antibody levels, $\mathrm{MN}$ titres and $\mathrm{ID}_{50}$ between two groups. The Spearman rank correlation coefficient was used for linear correlation analysis between the antibody levels and MN titres. All statistical tests were conducted using SPSS (version 20.0) for Windows (IBM). $P$ value less than 0.05 was considered statistically significant. The kinetics of anti-RBD IgG, anti-RBD IgM, anti-RBD IgA and MN titres during follow-up were calculated by the LOESS (locally estimated scatterplot smoothing) curve-fitting polynomial regression using $\mathrm{R}$ software (version 4.04).

Reporting Summary. Further information on research design is available in the Nature Research Reporting Summary linked to this article.

\section{Data availability}

The data supporting the findings of this study are available within the paper and the Supplementary Information. Source data are provided with this paper.

Received: 10 May 2021; Accepted: 16 December 2021; Published online: 7 February 2022

\section{References}

1. Zhu, N. et al. A novel coronavirus from patients with pneumonia in China, 2019. N. Engl. J. Med. 382, 727-733 (2020).

2. Tan, W. et al. A novel coronavirus genome identified in a cluster of pneumonia cases - Wuhan, China 2019-2020. China CDC Wkly. 2, 61-62 (2020).

3. Subramanian, R., He, Q. \& Pascual, M. Quantifying asymptomatic infection and transmission of COVID-19 in New York City using observed cases, serology, and testing capacity. Proc. Natl Acad. Sci. USA https://doi. org/10.1073/pnas.2019716118 (2021).

4. Mizumoto, K., Kagaya, K., Zarebski, A. \& Chowell, G. Estimating the asymptomatic proportion of coronavirus disease 2019 (COVID-19) cases on board the Diamond Princess cruise ship, Yokohama, Japan, 2020. Euro. Surveill. https://doi.org/10.2807/1560-7917.ES.2020.25.10.2000180 (2020).

5. Nishiura, H. et al. Estimation of the asymptomatic ratio of novel coronavirus infections (COVID-19). Int. J. Infect. Dis. 94, 154-155 (2020).

6. Oran, D. P. \& Topol, E. J. Prevalence of asymptomatic SARS-CoV-2 infection: a narrative review. Ann. Intern. Med. 173, 362-367 (2020).

7. Amanna, I. J., Carlson, N. E. \& Slifka, M. K. Duration of humoral immunity to common viral and vaccine antigens. N. Engl. J. Med. 357, 1903-1915 (2007)

8. Sariol, A. \& Perlman, S. Lessons for COVID-19 immunity from other coronavirus infections. Immunity 53, 248-263 (2020).

9. Edridge, A. W. D. et al. Seasonal coronavirus protective immunity is short-lasting. Nat. Med. 26, 1691-1693 (2020).

10. Liu, L. et al. Longitudinal profiles of immunoglobulin $\mathrm{G}$ antibodies against severe acute respiratory syndrome coronavirus components and neutralizing activities in recovered patients. Scand. J. Infect. Dis. 43, 515-521 (2011).

11. Cao, Z. et al. Potent and persistent antibody responses against the receptor-binding domain of SARS-CoV spike protein in recovered patients. Virol. J. 7, 299 (2010).

12. Choe, P. G. et al. MERS-CoV antibody responses 1 year after symptom onset, South Korea, 2015. Emerg. Infect. Dis. 23, 1079-1084 (2017).

13. Zhao, J. et al. Recovery from the Middle East respiratory syndrome is associated with antibody and T-cell responses. Sci. Immunol. https://doi. org/10.1126/sciimmunol.aan5393 (2017).
14. Seow, J. et al. Longitudinal observation and decline of neutralizing antibody responses in the three months following SARS-CoV-2 infection in humans. Nat. Microbiol. 5, 1598-1607 (2020).

15. Kim, Y. I. et al. Critical role of neutralizing antibody for SARS-CoV-2 reinfection and transmission. Emerg. Microbes Infect. 10, 152-160 (2021).

16. Chandrashekar, A. et al. SARS-CoV-2 infection protects against rechallenge in rhesus macaques. Science 369, 812-817 (2020).

17. Deng, W. et al. Primary exposure to SARS-CoV-2 protects against reinfection in rhesus macaques. Science 369, 818-823 (2020)

18. Weinreich, D. M. et al. REGN-COV2, a neutralizing antibody cocktail in outpatients with Covid-19. N. Engl. J. Med. 384, 238-251 (2021).

19. Chen, P. et al. SARS-CoV-2 neutralizing antibody LY-CoV555 in outpatients with Covid-19. N. Engl. J. Med. 384, 229-237 (2021).

20. Duan, K. et al. Effectiveness of convalescent plasma therapy in severe COVID-19 patients. Proc. Natl Acad. Sci. USA 117, 9490-9496 (2020).

21. Shen, C. et al. Treatment of 5 critically ill patients with COVID-19 with convalescent plasma. JAMA 323, 1582-1589 (2020).

22. Libster, R. et al. Early high-titer plasma therapy to prevent severe Covid-19 in older adults. N. Engl. J. Med. 384, 610-618 (2021).

23. Shi, D. et al. Dynamic characteristic analysis of antibodies in patients with COVID-19: a 13-month study. Front. Immunol. 12, 708184 (2021).

24. Hou, H. et al. Immunologic memory to SARS-CoV-2 in convalescent COVID-19 patients at 1 year postinfection. J. Allergy Clin. Immunol. https:// doi.org/10.1016/j.jaci.2021.09.008 (2021).

25. Zhang, J. et al. One-year sustained cellular and humoral immunities of COVID-19 convalescents. Clin. Infect. Dis. https://doi.org/10.1093/cid/ciab884 (2021)

26. Lau, E. H. Y. et al. Neutralizing antibody titres in SARS-CoV-2 infections. Nat. Commun. 12, 63 (2021)

27. Sterlin, D. et al. IgA dominates the early neutralizing antibody response to SARS-CoV-2. Sci. Transl. Med. https://doi.org/10.1126/scitranslmed.abd2223 (2021)

28. Gasser, R. et al. Major role of IgM in the neutralizing activity of convalescent plasma against SARS-CoV-2. Cell Rep. 34, 108790 (2021).

29. Antia, A. et al. Heterogeneity and longevity of antibody memory to viruses and vaccines. PLoS Biol. 16, e2006601 (2018).

30. Abu-Raddad, L. J. et al. SARS-CoV-2 antibody-positivity protects against reinfection for at least seven months with $95 \%$ efficacy. EClinicalMedicine $\mathbf{3 5}$ 100861 (2021)

31. Piccoli, L. et al. Mapping neutralizing and immunodominant sites on the SARS-CoV-2 spike receptor-binding domain by structure-guided high-resolution serology. Cell 183, 1024-1042.e1021 (2020).

32. Wang, Y. et al. Kinetics of viral load and antibody response in relation to COVID-19 severity. J. Clin. Invest. 130, 5235-5244 (2020).

33. Legros, V. et al. A longitudinal study of SARS-CoV-2-infected patients reveals a high correlation between neutralizing antibodies and COVID-19 severity. Cell Mol. Immunol. 18, 318-327 (2021)

34. Yang, Y. et al. Laboratory diagnosis and monitoring the viral shedding of SARS-CoV-2 infection. Innovation 1, 100061 (2020).

35. Gupta, V. et al. Asymptomatic reinfection in two healthcare workers from India with genetically distinct SARS-CoV-2. Clin. Infect. Dis. https://doi. org/10.1093/cid/ciaa1451 (2020).

36. To, K. K. et al. COVID-19 re-infection by a phylogenetically distinct SARS-coronavirus-2 strain confirmed by whole genome sequencing. Clin. Infect. Dis. https://doi.org/10.1093/cid/ciaa1275 (2020).

37. Van Elslande, J. et al. Symptomatic SARS-CoV-2 reinfection by a phylogenetically distinct strain. Clin. Infect. Dis. https://doi.org/10.1093/cid/ ciaa1330 (2020).

38. Letizia, A. G. et al. SARS-CoV-2 seropositivity and subsequent infection risk in healthy young adults: a prospective cohort study. Lancet Respir. Med. 9, 712-720 (2021)

39. Butt, A. A. et al. Outcomes among patients with breakthrough SARS-CoV-2 infection after vaccination: breakthrough SARS-CoV-2 infection. Int. J. Infect Dis. https://doi.org/10.1016/j.ijid.2021.08.008 (2021).

40. Hacisuleyman, E. et al. Vaccine breakthrough infections with SARS-CoV-2 variants. N. Engl. J. Med. 384, 2212-2218 (2021).

41. Kustin, T. et al. Evidence for increased breakthrough rates of SARS-CoV-2 variants of concern in BNT162b2-mRNA-vaccinated individuals. Nat. Med. 27, 1379-1384 (2021)

42. Hall, V. J. et al. SARS-CoV-2 infection rates of antibody-positive compared with antibody-negative health-care workers in England: a large, multicentre, prospective cohort study (SIREN). Lancet 397, 1459-1469 (2021).

43. Hansen, C. H., Michlmayr, D., Gubbels, S. M., Mølbak, K. \& Ethelberg, S. Assessment of protection against reinfection with SARS-CoV-2 among 4 million PCR-tested individuals in Denmark in 2020: a population-level observational study. Lancet 397, 1204-1212 (2021).

44. Khoury, D. S. et al. Neutralizing antibody levels are highly predictive of immune protection from symptomatic SARS-CoV-2 infection. Nat. Med. 27, 1205-1211 (2021). 
45. Sette, A. \& Crotty, S. Adaptive immunity to SARS-CoV-2 and COVID-19. Cell 184, 861-880 (2021).

46. Greaney, A. J. et al. Complete mapping of mutations to the SARS-CoV-2 spike receptor-binding domain that escape antibody recognition. Cell Host Microbe 29, 44-57.e49 (2021).

47. Korber, B. et al. Tracking changes in SARS-CoV-2 spike: evidence that D614G increases infectivity of the COVID-19 virus. Cell 182, 812-827.e819 (2020).

48. Baum, A. et al. Antibody cocktail to SARS-CoV-2 spike protein prevents rapid mutational escape seen with individual antibodies. Science 369, 1014-1018 (2020).

49. Greaney, A. J. et al. Comprehensive mapping of mutations in the SARS-CoV-2 receptor-binding domain that affect recognition by polyclonal human plasma antibodies. Cell Host Microbe https://doi.org/10.1016/j. chom.2021.02.003 (2021)

50. Thomson, E. C. et al. Circulating SARS-CoV-2 spike N439K variants maintain fitness while evading antibody-mediated immunity. Cell 184, 1171-1187 (2021)

51. Wibmer, C. K. et al. SARS-CoV-2 501Y.V2 escapes neutralization by South African COVID-19 donor plasma. Nat. Med. 27, 622-625 (2021).

52. Tegally, H. et al. Emergence and rapid spread of a new severe acute respiratory syndrome-related coronavirus 2 (SARS-CoV-2) lineage with multiple spike mutations in South Africa. Preprint at medRxiv https://doi. org/10.1101/2020.12.21.20248640 (2020).

53. Dupont, L. et al. Neutralizing antibody activity in convalescent sera from infection in humans with SARS-CoV-2 and variants of concern. Nat. Microbiol. 6, 1433-1442 (2021).

54. Supasa, P. et al. Reduced neutralization of SARS-CoV-2 B.1.1.7 variant by convalescent and vaccine sera. Cell 184, 2201-2211.e2207 (2021).

55. Zhou, D. et al. Evidence of escape of SARS-CoV-2 variant B.1.351 from natural and vaccine-induced sera. Cell https://doi.org/10.1016/j. cell.2021.02.037 (2021)

56. Wang, P. et al. Antibody resistance of SARS-CoV-2 variants B.1.351 and B.1.1.7. Nature https://doi.org/10.1038/s41586-021-03398-2 (2021).

57. Huang, B. et al. Neutralization of SARS-CoV-2 VOC 501Y.V2 by human antisera elicited by both inactivated BBIBP-CorV and recombinant dimeric RBD ZF2001 vaccines. Preprint at bioRxiv https://doi. org/10.1101/2021.02.01.429069 (2021).

58. Planas, D. et al. Reduced sensitivity of SARS-CoV-2 variant Delta to antibody neutralization. Nature 596, 276-280 (2021).

59. Lopez Bernal, J. et al. Effectiveness of Covid-19 vaccines against the B.1.617.2 (Delta) variant. N. Engl. J. Med. 385, 585-594 (2021)

60. Muecksch, F. et al. Affinity maturation of SARS-CoV-2 neutralizing antibodies confers potency, breadth, and resilience to viral escape mutations. Immunity 54, 1853-1868.e1857 (2021).

61. Sokal, A. et al. Maturation and persistence of the anti-SARS-CoV-2 memory B cell response. Cell 184, 1201-1213.e1214 (2021).

62. Wang, Z. et al. Naturally enhanced neutralizing breadth against SARS-CoV-2 one year after infection. Nature 595, 426-431 (2021).

63. Dejnirattisai, W. et al. Antibody evasion by the P.1 strain of SARS-CoV-2. Cell 184, 2939-2954.e2939 (2021).

64. Robbiani, D. F. et al. Convergent antibody responses to SARS-CoV-2 in convalescent individuals. Nature 584, 437-442 (2020).

65. Leidi, A. et al. Risk of reinfection after seroconversion to SARS-CoV-2: a population-based propensity-score matched cohort study. Clin. Infect. Dis. https://doi.org/10.1093/cid/ciab495 (2021).

66. Pegu, A. et al. Durability of mRNA-1273 vaccine-induced antibodies against SARS-CoV-2 variants. Science https://doi.org/10.1126/science.abj4176 (2021).

67. Widge, A. T. et al. Durability of responses after SARS-CoV-2 mRNA-1273 vaccination. N. Engl. J. Med. 384, 80-82 (2021).

68. Krammer, F. et al. Antibody responses in seropositive persons after a single dose of SARS-CoV-2 mRNA vaccine. N. Engl. J. Med. 384, 1372-1374 (2021).

69. Demonbreun, A. R. et al. Comparison of IgG and neutralizing antibody responses after one or two doses of COVID-19 mRNA vaccine in previously infected and uninfected individuals. EClinicalMedicine 38, 101018 (2021).

70. Manisty, C. et al. Antibody response to first BNT162b2 dose in previously SARS-CoV-2-infected individuals. Lancet 397, 1057-1058 (2021).
71. Yang, Y. et al. Plasma IP-10 and MCP-3 levels are highly associated with disease severity and predict the progression of COVID-19. J. Allergy Clin. Immunol. 146, 119-127.e114 (2020).

72. Liu, Y. X. et al. Elevated plasma levels of selective cytokines in COVID-19 patients reflect viral load and lung injury. Natl Sci. Rev. 7, 1003-1011 (2020).

73. Long, Q. X. et al. Clinical and immunological assessment of asymptomatic SARS-CoV-2 infections. Nat. Med. 26, 1200-1204 (2020).

74. Liu, C. et al. The architecture of inactivated SARS-CoV-2 with postfusion spikes revealed by cryo-EM and cryo-ET. Structure 28, 1218-1224.e1214 (2020).

75. Yang, Y. et al. Development of a quadruple qRT-PCR assay for simultaneous identification of highly and low pathogenic H7N9 avian influenza viruses and characterization against oseltamivir resistance. BMC Infect. Dis. 18, 406 (2018).

76. Yang, Y. et al. Development of a reverse transcription quantitative polymerase chain reaction-based assay for broad coverage detection of African and Asian Zika virus lineages. Virol. Sin. 32, 199-206 (2017).

77. Reed, L. \& Muench, H. A simple method for estimating fifty percent endpoints. Am. J. Hyg. 27, 493-497 (1938).

78. Reynolds, C. J. et al. Discordant neutralizing antibody and T cell responses in asymptomatic and mild SARS-CoV-2 infection. Sci. Immunol. https://doi. org/10.1126/sciimmunol.abf3698 (2020).

79. Wu, F. et al. Evaluating the association of clinical characteristics with neutralizing antibody levels in patients who have recovered from mild COVID-19 in Shanghai, China. JAMA Intern. Med. 180, 1356-1362 (2020).

80. To, K. K. et al. Temporal profiles of viral load in posterior oropharyngeal saliva samples and serum antibody responses during infection by SARS-CoV-2: an observational cohort study. Lancet Infect. Dis. 20, 565-574 (2020).

81. Wajnberg, A. et al. Robust neutralizing antibodies to SARS-CoV-2 infection persist for months. Science 370, 1227-1230 (2020).

82. $\mathrm{Wu}, \mathrm{Y}$. et al. A noncompeting pair of human neutralizing antibodies block COVID-19 virus binding to its receptor ACE2. Science 368, 1274-1278 (2020).

\section{Acknowledgements}

This work was supported by grants from National Natural Science Foundation of China (32170936), National Science and Technology Major Project (2021YFC0863300, 2021YFC2301800). We thank the biological sample bank of the Shenzhen Third People's Hospital for bio-samples and services provided.

\section{Author contributions}

Y. Liu, J.Y. and Y.Y. designed the study. Y.Y., M.Y., L.G. and Y.P. performed the neutralization assays and contributed to the analysis and interpretation of data. Y. Liang, J.W., L.X., X.L., J.L., J.W., M.L., Z.X., M.Z. and F.W. enrolled the patients and collected the clinical data. Y.Y. and M.Y. drafted the article. Y.S. critically revised important intellectual content. All authors agree to be accountable for the content of the work.

\section{Competing interests}

The authors declare no competing interests.

\section{Additional information}

Extended data is available for this paper at https://doi.org/10.1038/s41564-021-01051-2. Supplementary information The online version contains supplementary material available at https://doi.org/10.1038/s41564-021-01051-2.

Correspondence and requests for materials should be addressed to Jing Yuan or Yingxia Liu.

Peer review information Nature Microbiology thanks Jincun Zhao and the other, anonymous, reviewer(s) for their contribution to the peer review of this work.

Reprints and permissions information is available at www.nature.com/reprints.

Publisher's note Springer Nature remains neutral with regard to jurisdictional claims in published maps and institutional affiliations.

(c) The Author(s), under exclusive licence to Springer Nature Limited 2022 
A

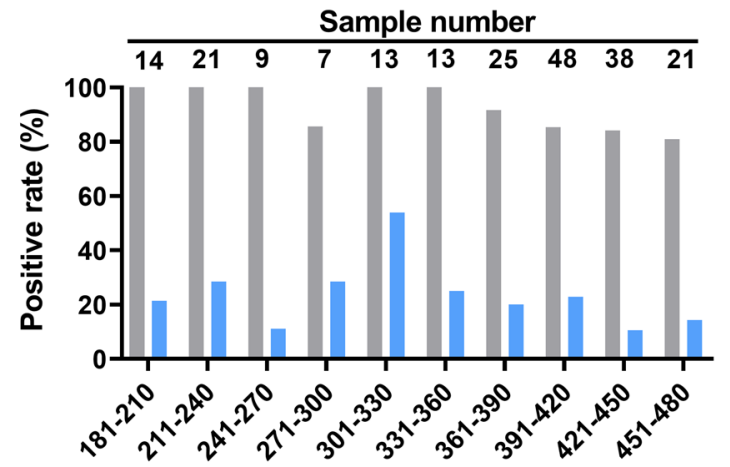

Days post illness onset/laboratory confirmation Anti-RBD IgA Anti-RBD IgM
B

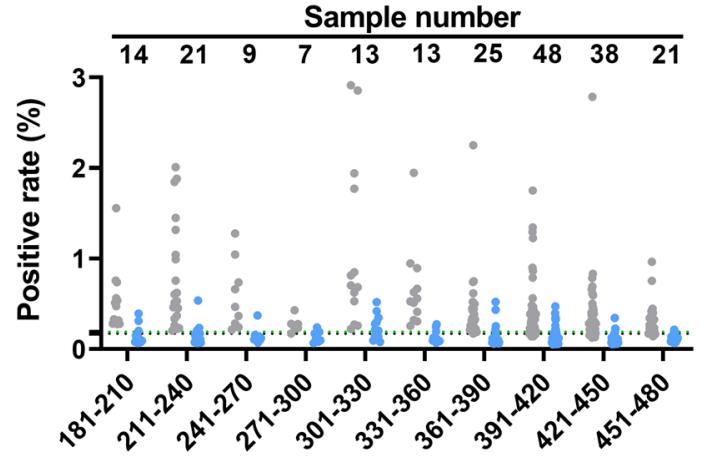

Days post illness onset/laboratory confirmation

- Anti-RBD IgA - Anti-RBD IgM

C
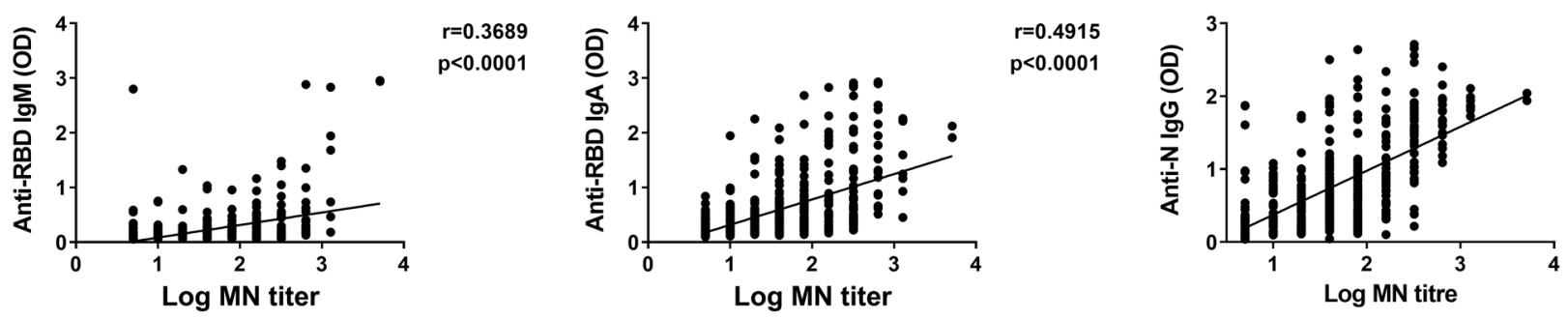

$r=0.5996$ $\mathrm{p}<0.0001$

D
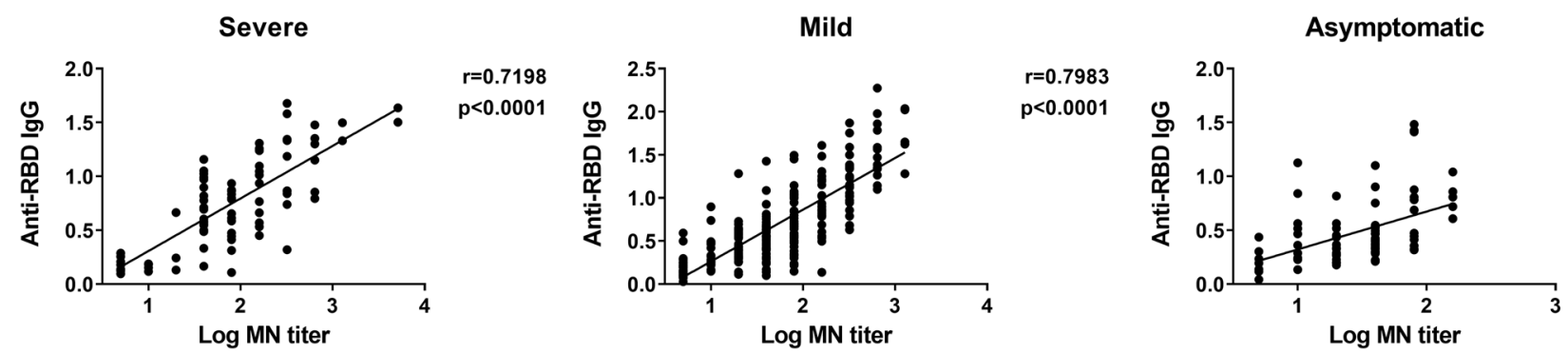

Extended Data Fig. 1 | Persistent antibody responses in COVID-19 convalescents. (A) and (B) Positive rates and detailed OD values of the anti-RBD IgM and anti-RBD IgA for the COVID-19 convalescents with over 180 days follow-up. (C) Spearman correlation plot between the OD values of anti-RBD IgM $(p<0.001)$, anti-RBD IgA $(p<0.001)$ and anti-N IgG $(p<0.001)$ with the log MN titers. (D) Spearman correlation plot of log MN titers with anti-RBD IgG in COVID-19 patients with different clinical spectrum $(p<0.001)$. Specimens with MN titers less than 10 were assigned a value of 5 . Dotted lines indicate the limit of detection. 
A

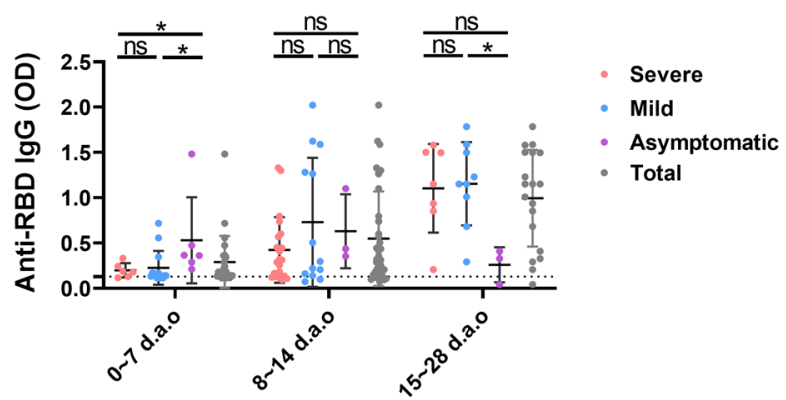

B

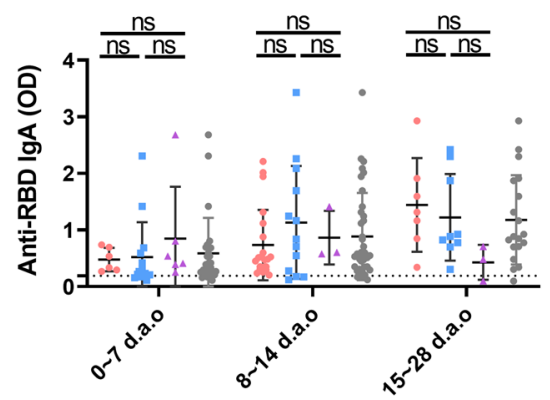

- Severe

- Mild

Asymptomatic

- Total

C

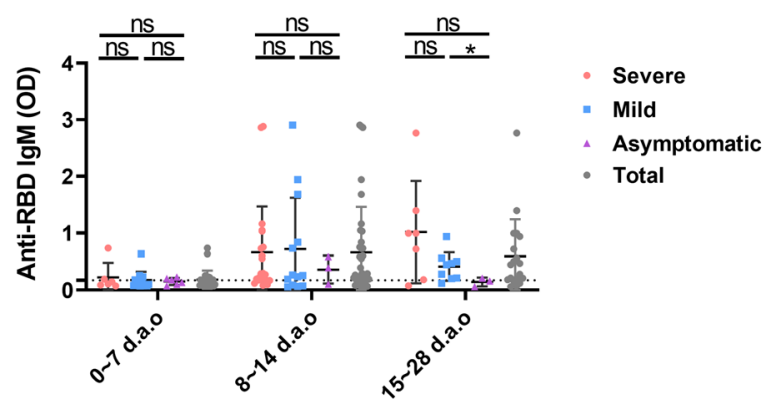

D

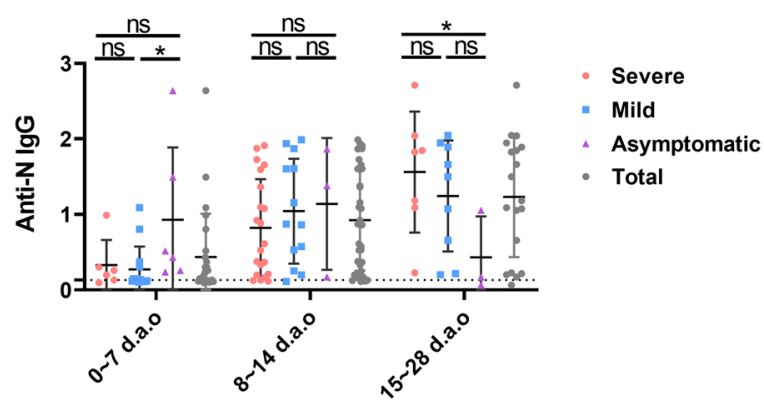

E

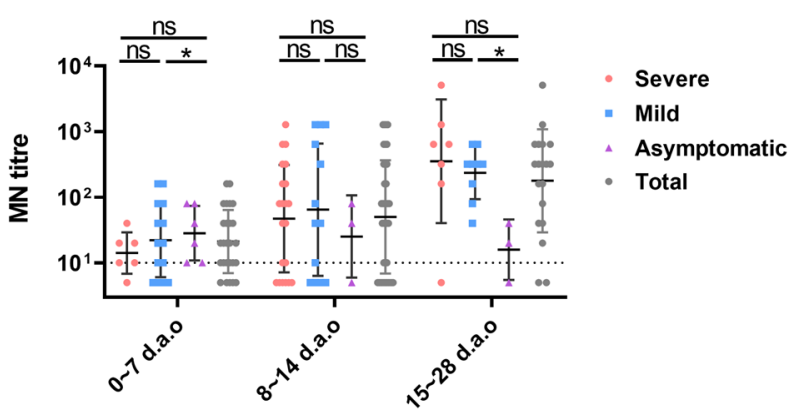

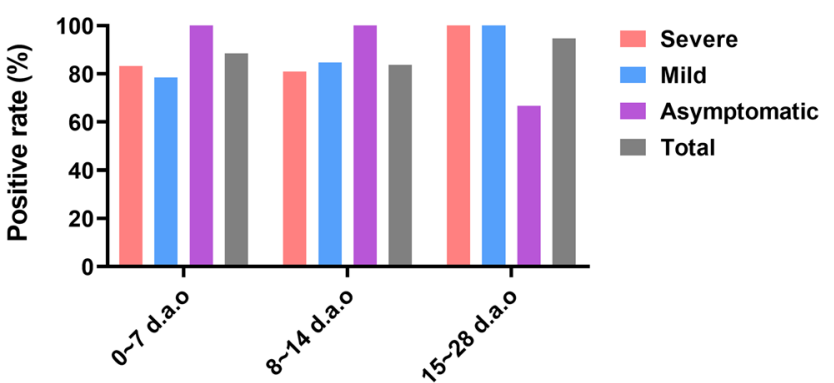
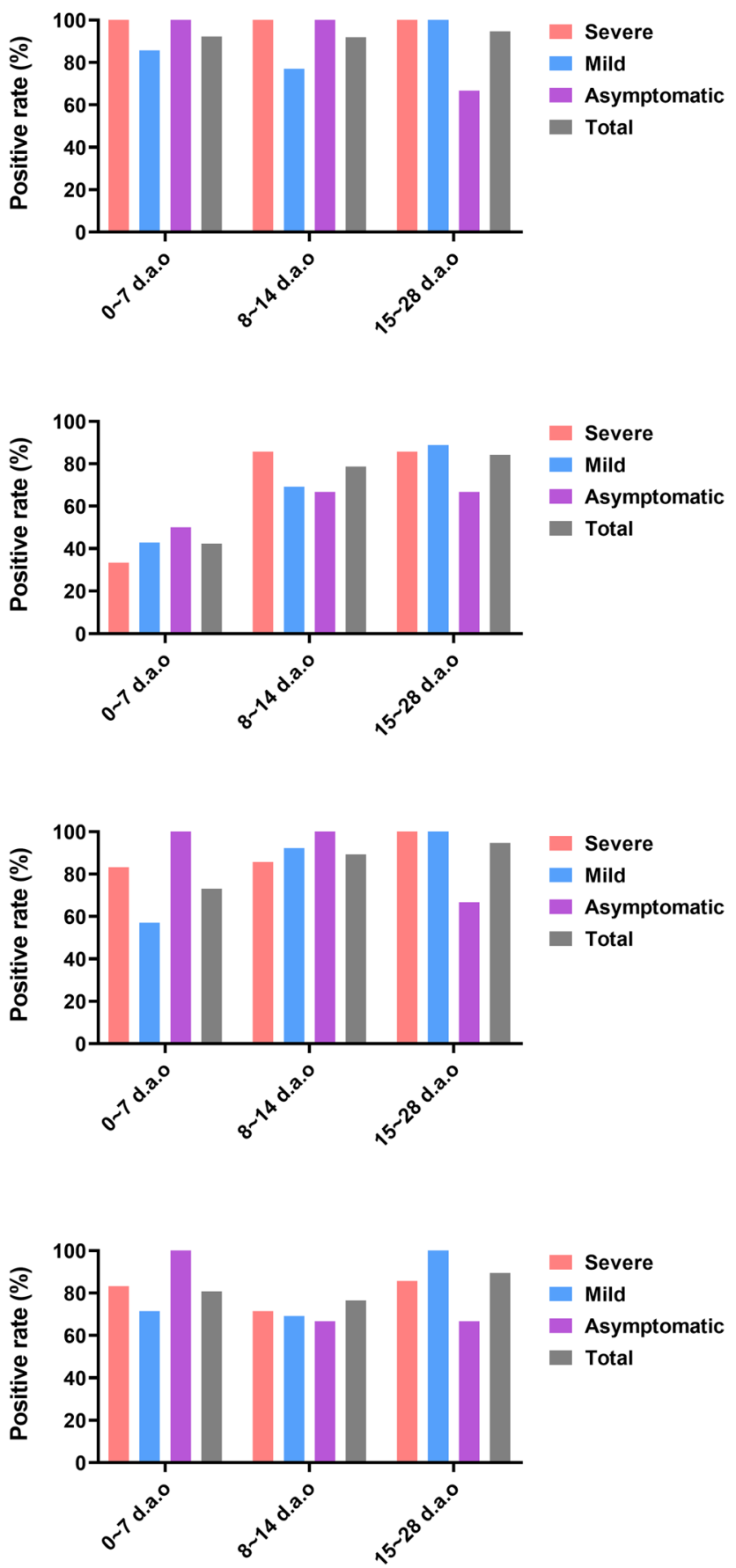

Extended Data Fig. 2 | See next page for caption. 
Extended Data Fig. 2 | Comparison of the antibody responses of COVID-19 patients with different clinical spectrum within 28 d.a.o. The positive rates and exact values of anti-RBD IgG (A), anti-RBD IgA (B), anti-RBD IgM (C), anti-N IgG (D) and MN titers (E). Statistical significance was measured using a Mann-Whitney two-sided test $\mathrm{U}$-test. ${ }^{*} \mathrm{P}<0.05,{ }^{* \star} \mathrm{P}<0.01,{ }^{\star \star \star} \mathrm{P}<0.001$. ns, not significant. The line and error bar represent the mean and standard deviation for the anti-RBD IgG, while geometric mean titers and standard deviation for the MN titers. Black dotted lines indicate the limit of detection for anti-RBD $\lg M$, and green dotted lines indicate the limit of detection for anti-RBD IgA. 
A

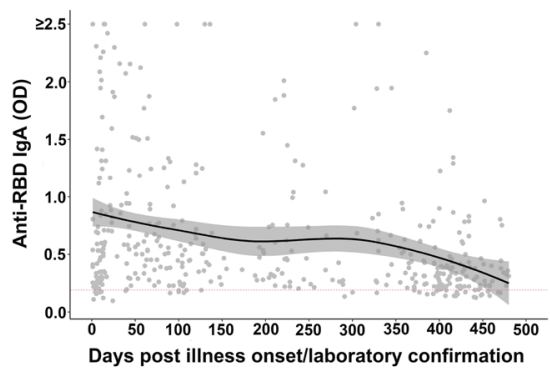

D

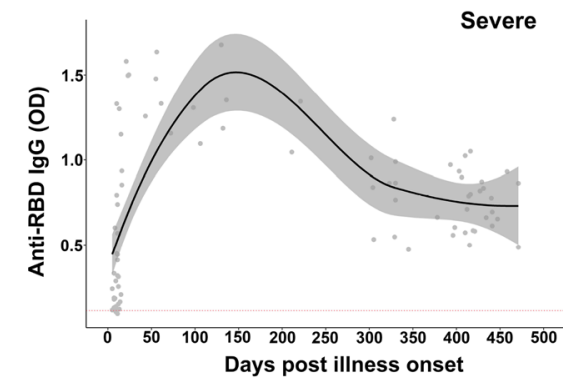

E

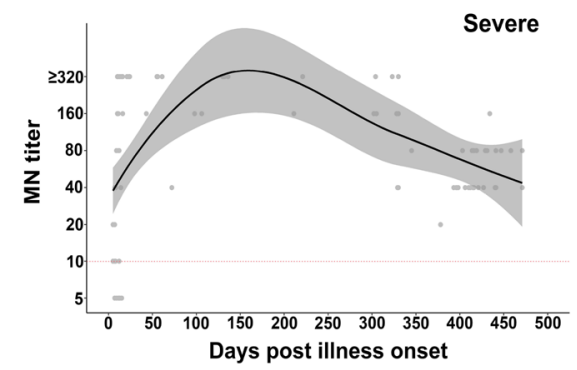

B
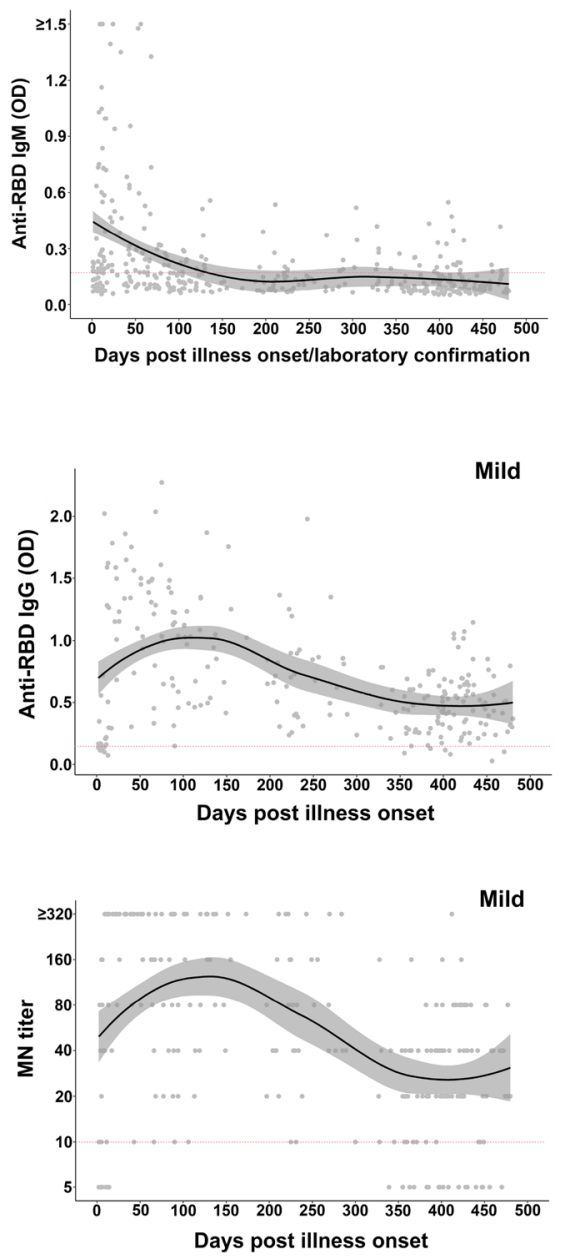

C
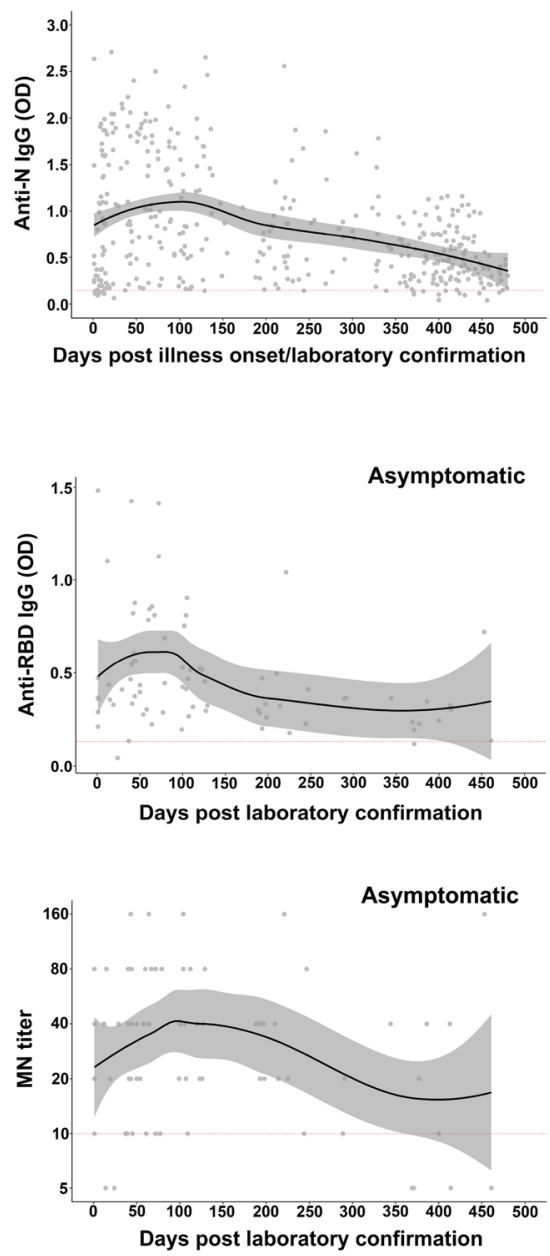

Extended Data Fig. 3 | Kinetics of SARS-CoV-2 specific antibody responses in COVID-19 convalescents during the 480 days follow-up. (A), (B) and (C) Kinetics of anti-RBD IgA, anti-RBD IgM and anti-N IgG during the 480 days follow-up. (D) and (E) Kinetics of anti-RBD IgG and MN titers of the COVID-19 patients with different clinical spectrum during the 480 days follow-up. The black solid line represents the fitted curve obtained using the LOESS (locally estimated scatterplot smoothing) curve fitting polynomial regression. Gray band areas represent $95 \%$ confidence intervals. Specimens with OD values $\geq 2.5$ were assigned a value of 2.5 for anti-RBD IgA, and OD values $\geq 1.5$ were assigned a value of 1.5 for anti-RBD IgM. MN titers $\geq 320$ were assigned a value of 320. Dotted lines indicate the limit of detection. 


\section{nature portfolio}

Corresponding author(s): Yingxia Liu and Jing Yuan

Last updated by author(s): Dec 11, 2021

\section{Reporting Summary}

Nature Portfolio wishes to improve the reproducibility of the work that we publish. This form provides structure for consistency and transparency in reporting. For further information on Nature Portfolio policies, see our Editorial Policies and the Editorial Policy Checklist.

\section{Statistics}

For all statistical analyses, confirm that the following items are present in the figure legend, table legend, main text, or Methods section.

n/a Confirmed

$\bigotimes$ The exact sample size $(n)$ for each experimental group/condition, given as a discrete number and unit of measurement

$\triangle$ A statement on whether measurements were taken from distinct samples or whether the same sample was measured repeatedly

The statistical test(s) used AND whether they are one- or two-sided

Only common tests should be described solely by name; describe more complex techniques in the Methods section.

$\triangle \square$ A description of all covariates tested

Х $\square$ A description of any assumptions or corrections, such as tests of normality and adjustment for multiple comparisons

$\square$ A full description of the statistical parameters including central tendency (e.g. means) or other basic estimates (e.g. regression coefficient)

AND variation (e.g. standard deviation) or associated estimates of uncertainty (e.g. confidence intervals)

$\triangle$ For null hypothesis testing, the test statistic (e.g. $F, t, r$ ) with confidence intervals, effect sizes, degrees of freedom and $P$ value noted

Give $P$ values as exact values whenever suitable.

Х $\square$ For Bayesian analysis, information on the choice of priors and Markov chain Monte Carlo settings

Х $\square$ For hierarchical and complex designs, identification of the appropriate level for tests and full reporting of outcomes

$\square$ Estimates of effect sizes (e.g. Cohen's $d$, Pearson's $r$ ), indicating how they were calculated

Our web collection on statistics for biologists contains articles on many of the points above.

\section{Software and code}

Policy information about availability of computer code

Data collection No software was used for data collection.

Data analysis We used SPSS 20.0 for Windows, GraphPad Prism 8.0 and R software 4.04 for the analysis of the data.

For manuscripts utilizing custom algorithms or software that are central to the research but not yet described in published literature, software must be made available to editors and

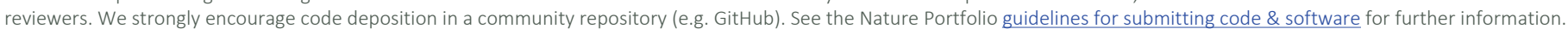

\section{Data}

Policy information about availability of data

All manuscripts must include a data availability statement. This statement should provide the following information, where applicable:

- Accession codes, unique identifiers, or web links for publicly available datasets

- A description of any restrictions on data availability

- For clinical datasets or third party data, please ensure that the statement adheres to our policy 
Please select the one below that is the best fit for your research. If you are not sure, read the appropriate sections before making your selection.

\ Life sciences

Behavioural \& social sciences

$\square$ Ecological, evolutionary \& environmental sciences

For a reference copy of the document with all sections, see nature.com/documents/nr-reporting-summary-flat.pdf

\section{Life sciences study design}

All studies must disclose on these points even when the disclosure is negative.

$\begin{array}{ll}\text { Sample size } & \begin{array}{l}\text { A total of } 214 \text { laboratory confirmed COVID-19 patients admitted to our hospital during January } 11 \text { to April } 18,2020 \text { were followed up and } \\ \text { included in our analysis. The sample size was based on availability of serum samples collected at multiple time points from patients admitted } \\ \text { to our hospital. No prior sample size calculation was performed. }\end{array} \\ \text { Data exclusions } & \text { No data excluded. } \\ \text { Replication } & \begin{array}{l}\text { For the viral entry inhibition assay with SARS-CoV-2 pseudotyped virus and authentic SARS-CoV-2 microneutralization (MN) assays, all the } \\ \text { measurements were performed in triplicate. We confirm that all attempts at replication were successful. }\end{array} \\ \text { Randomization } & \text { Randomization was not relevant to this study as this was an observational study. } \\ \text { Blinding } & \text { Blinding was not relevant to this study because of the observational design. }\end{array}$

\section{Reporting for specific materials, systems and methods}

We require information from authors about some types of materials, experimental systems and methods used in many studies. Here, indicate whether each material, system or method listed is relevant to your study. If you are not sure if a list item applies to your research, read the appropriate section before selecting a response.

\begin{tabular}{l|l} 
Materials \& experimental systems \\
\hline n/a & Involved in the study \\
$\square$ & $\bigotimes$ Antibodies \\
$\square$ & $\square$ Eukaryotic cell lines \\
$\square$ & $\square$ Animals and other organisms \\
$\square$ & $\square$ Human research participants \\
$\triangle$ & $\square$ Clinical data \\
$\square$ & $\square$ Dual use research of concern
\end{tabular}

\begin{tabular}{l} 
Methods \\
\hline n/a \\
$\square$ \\
$\square$ Involved in the study \\
$\square$ \\
$\square$ ChIP-seq \\
$\square$ Flow cytometry \\
$\square$ MRI-based neuroimaging
\end{tabular}

Antibodies

Antibodies used

The used SARS-CoV-2 RBD specific antibody B38 was previously isolated from a COVID-19 patient by our group, and it has been proved to significantly neutralize wild type SARS-CoV-2 (Wu, Y. et al. A noncompeting pair of human neutralizing antibodies block COVID-19 virus binding to its receptor ACE2. Science 368, 1274-1278).

Validation

The data of this antibody was published in Science ( $\mathrm{Wu}, \mathrm{Y}$. et al. A noncompeting pair of human neutralizing antibodies block COVID-19 virus binding to its receptor ACE2. Science 368, 1274-1278).

\section{Eukaryotic cell lines}

Policy information about cell lines

Cell line source(s)

African green monkey kidney Vero cell (ATCC, CCL-81) were obtained from ATCC, and 293 cells stably expressing ACE-2 (293ACE-2) were obtained from Vazyme (Vazyme).

Authentication

None of the cell lines used were authenticated.

Mycoplasma contamination

All cell lines used were tested (by PCR) and were mycoplasma free.

Commonly misidentified lines

(See ICLAC register)

No commonly misidentified lines were used in this study. 
Human research participants

Policy information about studies involving human research participants

Population characteristics

Recruitment

Ethics oversight
A total of 214 laboratory confirmed COVID-19 patients with 51 individuals in severe group, 134 individuals in mild group and 29 individuals in asymptomatic group were included in this study for the analysis of antibody response. This cohort was $46.73 \%$ male with an average age of 48 years (range 2-79).

No participants were enrolled. All samples preexisted.

The study protocol was approved by the Ethics Committees of Shenzhen Third People's Hospital (2020-010).

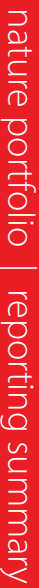

Note that full information on the approval of the study protocol must also be provided in the manuscript.

3 